\title{
SOME GEOMETRIC PROPERTIES ON THE FOURIER AND FOURIER STIELTJES ALGEBRAS OF LOCALLY COMPACT GROUPS, ARENS REGULARITY AND RELATED PROBLEMS
}

\author{
ANTHONY TO-MING LAU AND ALI ÜLGER
}

\begin{abstract}
Let $G$ be a locally compact topological group and $A(G) \quad[B(G)]$ be, respectively, the Fourier and Fourier-Stieltjes algebras of $G$. It is one of the purposes of this paper to investigate the RNP (= Radon-Nikodym property) and some other geometric properties such as weak RNP, the Dunford-Pettis property and the Schur property on the algebras $A(G)$ and $B(G)$, and to relate these properties to the properties of the multiplication operator on the group $C^{*}$ algebra $C^{*}(G)$. We also investigate the problem of Arens regularity of the projective tensor products $C^{*}(G) \hat{\otimes} A$, when $B(G)=C^{*}(G)^{*}$ has the RNP and $A$ is any $C^{*}$-algebra. Some related problems on the measure algebra, the group algebra and the algebras $A_{p}(G), P F_{p}(G), P M_{p}(G) \quad(1<p<\infty)$ are also discussed.
\end{abstract}

\section{INTRODUCTION}

Let $G$ be a locally compact group and $A(G)[B(G)]$ be the Fourier and Fourier-Stieltjes algebras of $G$, respectively. In [34] E. Granirer and M. Leinert, answering a conjecture of McKennon [52], proved that, for any locally compact group $G$, on the unit sphere $S=\{\phi \in B(G):\|\phi\|=1\}$, the weak*-topology and the multiplier topology agree. The multiplier topology is the one for which a net $\left(\phi_{\alpha}\right)$ converges to $\phi$ if and only if, for each $\psi \in A(G),\left\|\left(\phi_{\alpha}-\phi\right) \psi\right\| \rightarrow 0$. In particular, if the group $G$ is compact, on the unit sphere $S$ of $B(G)(=A(G))$ the weak* and the normal topologies agree so that the space $A(G)$ has the RNP (= Radon-Nikodym property) [6, Theorem 4.4.3]. In [66] K. Taylor showed that the compact groups are not the only topological groups for which the space $A(G)$ has the RNP. For instance, if $G$ is the Fell group, which is not compact, then the space $A(G)$ has the RNP as well. One of the purposes of this paper is to further investigate the RNP and some other geometric properties such as weak RNP, the DPP (= Dunford-Pettis property) and the Schur property on the algebras $A(G)$ and $B(G)$, and to relate these properties to the properties of the multiplication operator $\tau_{f}$ on the group $C^{*}$-algebra $C^{*}(G)$. Here, for $f$ in $C^{*}(G)$, the

Received by the editors February 11, 1991.

1980 Mathematics Subject Classification (1985 Revision). Primary 43A10, 43A30, 43A60, 46B22, 46H99, 46M05, 22D25; Secondary 47C15, 46H20.

Key words and phrases. Locally compact groups, amenability, Fourier and Fourier-Stieltjes algebras, group algebra, measure algebra, group $C^{*}$-algebra, multiplier algebra, regular representation, Arens regularity, Radon-Nikodym property, Dunford-Pettis property, Schur property. 
operator $\tau_{f}: C^{*}(G) \rightarrow C^{*}(G)$ is defined by $\tau_{f}(g)=f \cdot g$, the product of $f$ and $g$ in $C^{*}(G)$. Some of the results of the paper can be summarized as follows (see Theorems 4.3 and 4.5): For any locally compact group $G$, the following are equivalent. (a) $G$ is compact. (b) The space $B(G)$ has the RNP and the DPP. (c) The space $B(G)$ is an $l^{1}$-sum of finite dimensional Banach spaces. (d) The space $B(G)$ has the Schur property. (e) The $C^{*}$ algebra $C^{*}(G)$ is scattered and has the DPP. (f) The algebra $C^{*}(G)$ does not contain an isomorphic copy of $l^{1}$ and has the DPP. (g) Every bounded linear operator $T: C^{*}(G) \rightarrow B(G)$ is compact. (h) Each functional $\phi$ in $B(G)$ is almost periodic on $C^{*}(G)$. (i) For each $f$ in $C^{*}(G)$, the multiplication operator $\tau_{f}: C^{*}(G) \rightarrow C^{*}(G) \quad\left(\tau_{f}(g)=f \cdot g\right)$ is compact. (j) For each $f$ in $C^{*}(G)$, the multiplication operator $\tau_{f}$ is weakly compact. (k) The $C^{*}$-algebra $C^{*}(G)$ is a $c_{0}$-sum of finite dimensional $C^{*}$-algebras. (l) The von Neumann algebra $V N(G)$ generated by the left regular representation of $G$ is the direct summand of finite dimensional $C^{*}$-algebras. (m) The unit ball of $V N(G)$ has jointly continuous multiplication in the $\sigma$ (= ultraweak) topology. (n) $G$ is an [IN]-group and the space $A(G)$ has the RNP. (o) Each functional $\phi$ in $A(G)$ is almost periodic on $V N(G)$. (p) $G$ is a [MOORE]-group and $B(G)$ has the RNP. We also show that (Theorem 6.7) the locally compact group $G$ is an [AU]-group (i.e., the enveloping von Neumann algebra of $C^{*}(G)$ is atomic [66, Theorem 4.2]) if and only if, for each $f$ in $C^{*}(G)$, the multiplication operator $\tau_{f}$ from $C^{*}(G)$ into $C^{*}(G)$ is a weakly precompact operator. That is, for each bounded sequence $\left(g_{n}\right)$ in $C^{*}(G)$, the sequence $\left(f \cdot g_{n}\right)$ has a weakly Cauchy subsequence. This result draws a fine line between compact groups (for which $\tau_{f}$ is weakly compact) and [AU]-groups. At this point we note that if $G$ is the Fell group [3] for instance then, for every $f$ in $C^{*}(G)$, the operator $\tau_{f}$ is weakly precompact but, $G$ not being compact, $\tau_{f}$ is not weakly compact for at least one $f$ in $C^{*}(G)$.

Another problem tackled in the paper is the problem of Arens regularity of the projective tensor product of two $C^{*}$-algebras. As is well known [2] on the second dual $A^{* *}$ of any Banach algebra $A$ there exist two algebra multiplications extending that of $A$. When these multiplications coincide on $A^{* *}$ then the algebra $A$ is said to be Arens regular. Given any two Banach algebras $A$ and $B$, on their projective tensor product $A \hat{\otimes} B$ there exists a natural Banach algebra multiplication which is the linear extension of the following multiplication on decomposable tensors

$$
a \otimes b \cdot \tilde{a} \otimes \tilde{b}=a \tilde{a} \otimes b \tilde{b}
$$

see Chapter VI of the book [5] and the papers [27 and 68]. The Arens regularity of the algebra $A \hat{\otimes} B$ has been studied in [71] where it has been shown, among other things, that the projective tensor product of two commutative $C^{*}$-algebras is Arens regular. The question whether the projective tensor product of two noncommutative $C^{*}$-algebras is Arens regular or not was left open. The results we present in this paper show that the projective tensor product of even two von Neumann algebras need not be Arens regular and that, for two von Neumann algebras $A$ and $B$, Arens regularity of the algebra $A \hat{\otimes} B$ is closely connected with the properties that the algebra $A$ or $B$ is finite or atomic. In particular we shall see that, for any locally compact group $G, G$ is compact if and only if the space $B(G)$ (resp. $A(G)$ ) has the RNP and for any $C^{*}$-algebra $A$, the alge- 
bra $C^{*}(G) \hat{\otimes} A$ (resp. $C_{\rho}^{*}(G) \hat{\otimes} A$ ) is Arens regular. The fact that the projective tensor product of two $C^{*}$-algebras need not be Arens regular also solves in the negative the following two questions that arise naturally. To explain these two questions, let $A$ and $B$ be two $C^{*}$-algebras and $A \otimes B$ be their algebraic tensor product. Although the space $A \otimes B$ is a *-algebra, the projective tensor norm is not a $C^{*}$-algebra norm. However there always exists a cross norm on $A \otimes B$ under which the completion of $A \otimes B$ is a $C^{*}$-algebra [60, p. 60]. Another alternative that comes to one's mind is this: Can we keep the projective tensor norm but change the multiplication on $A \hat{\otimes} B$ so as to make it into a $C^{*}$-algebra? Arens regularity considerations will show that this is not possible. To explain the second question, we recall that according to Grothendieck-Pisier-Haagerup inequality [38], every bounded linear operator from $A$ to $B^{*}$ (or, from $B$ to $A^{*}$ ) is weakly compact. This is of course not a characteristic property of $C^{*}$ algebras and the following question arises naturally: Is every bounded linear operator $u: A \hat{\otimes} B \rightarrow(A \hat{\otimes} B)^{*}$ weakly compact? Again Arens regularity consideration will show that this is not the case. Some other applications to related problems are also given.

Arens regularity of the commutative Banach algebra $A_{p}(G), 1<p<\infty$, has recently been studied by B. Forest [26]. It is shown, for example, that if the algebra $A_{p}(G)$ is Arens regular then the group $G$ is discrete. Furthermore, as shown in [51, Proposition 5.3], for $p=2$ and $G$ amenable, $A_{p}(G)=$ $A(G)$ is Arens regular if and only if $G$ is finite. In this paper, we shall study some problems related to Arens regularity of the algebra $A_{p}(G)$. We shall also establish a one-to-one correspondence between the compact subgroups of $G$ and all nonzero $\sigma\left(A_{p}(G), L^{1}(G)\right)$-closed right translation invariant subalgebras of $A_{p}(G)$ which are closed under conjugation and a one-to-one correspondence between the set of closed subgroups of $G$ and certain topologically invariant subalgebras of $P M_{p}(G)$.

This paper is organized as follows. In $\S 2$ we gather some notations and definitions we need in the sequel. In $\S 3$ we establish some general results on the geometry of the dual space of a $C^{*}$-algebra. We answer in particular a problem raised by Professor John Duncan during the regional conference of the American Mathematical Society held in Fayetteville (Arkansas), March 1990, concerning the characterization of those $C^{*}$-algebras $A$ on which each functional $f^{*}$ in $A^{*}$ is almost periodic. In $\S 4$, we apply the characterizations obtained in $\S 3$ to our main results in $\S 4$ concerning geometric properties of the algebras $A(G)$ and $B(G)$. In $\S 5$ we study similar properties on the group algebra $L^{1}(G)$ and the measure algebra $M(G)$. In $\S 6$, we give some results relating the properties of the group $G$ to those of the multiplication operator $\tau_{f}$ on $C^{*}(G)$. In $\S 7$ we study Arens regularity of the projective tensor products $C^{*}(G) \hat{\otimes} A$ and $C_{\rho}^{*}(G) \hat{\otimes} A$, when the space $B(G)$ (resp. $A(G)$ ) has the RNP and $A$ is a $C^{*}$-algebra. We also answer some questions raised in [71] as well as some other related problems. Finally, in $\S 8$ we study Arens regularity and invariant subalgebras of the Banach algebra $A_{p}(G)$ as well as certain weak*-closed topologically invariant subspaces of $P M_{p}(G)$.

\section{Preliminaries AND SOME NOTATIONS}

Let $G$ be a locally compact group with a fixed left Haar measure $\mu$, which is also denoted by $d x$. The usual $L^{p}(G)$ spaces $(1 \leq p \leq \infty)$ used in this 
paper are defined with respect to this measure $\mu$. By $C(G)$ we denote the Banach space of bounded continuous complex valued functions endowed with the supremum norm. By $C_{0}(G)$ we denote the subspace of $C(G)$ consisting of the functions which are null at infinity. For any Banach space $X$, we denote by $X^{*}$ and $X^{* *}$ its first and second continuous duals. We regard $X$ as naturally embedded into $X^{* *}$. For $x$ in $X$ and $x^{*}$ in $X^{*}$, by $\left\langle x, x^{*}\right\rangle$ (or by $\left\langle x^{*}, x\right\rangle$ ) we denote the natural duality between $X$ and $X^{*}$. For any Banach algebra $A, a$ in $A$ and $f$ in $A^{*}$, by $a f$ (resp. $f a$ ) we denote the element of $A^{*}$ defined by $\langle a f, b\rangle=\langle f, b a\rangle$ (resp. $\langle f a, b\rangle=\langle f, a b\rangle$ ). Also, for $f$ in $C(G)$ and $s$ in $G, s f$ is the element of $C(G)$ defined by $s f(t)=f(s t)$. The group $G$ is said to be "amenable" if there exists a positive functional on $C(G)$ of norm one such that $\phi(s f)=\phi(f)$ for all $f$ in $C(G)$ and $s$ in $G$. All solvable groups and all compact groups are known to be amenable. However, the free group on two generators is not amenable. For more information on this subject we refer the reader to Greenleaf's book [36] and the recent books of Pier [56] and Paterson [54]. As usual, we denote by $C^{*}(G)$ the group $C^{*}$-algebra of $G$ [25] and by $C_{\rho}^{*}(G)$ the reduced group $C^{*}$-algebra of $G$. That is, $C_{\rho}^{*}(G)$ is the norm closure of the space $\left\{\rho(f): f \in L^{1}(G)\right\}$ in the operator algebra $B\left(L^{2}(G)\right)$ of bounded linear operators on $L^{2}(G)$. Here $\rho(f): L^{2}(G) \rightarrow L^{2}(G)$ is the linear operator defined by $\rho(f)(g)=f * g$, the convolution of the functions $f$ and $g$. If we denote the norm of an element $f$ in $C^{*}(G)$ by $\|f\|$ then one always has $\|f\| \geq\|\rho(f)\|$ for each $f$ in $L^{1}(G)$, where $\|\rho(f)\|$ is the operator norm of $\rho(f)$ as an element of $B\left(L^{2}(G)\right)$. These two norms are equal if and only if the group $G$ is amenable [36, p. 161], and in this case the two $C^{*}$-algebras $C^{*}(G)$ and $C_{\rho}^{*}(G)$ coincide.

Let $P(G)$ denote the subspace of $C(G)$ consisting of all positive definite functions on $G$, and let $B(G)$ be its linear span. Then, as is well known [25], the space $B(G)$ can be identified with the dual of the algebra $C^{*}(G)$. In this identification, the elements of $P(G)$ correspond precisely to the positive linear functionals on $C^{*}(G)$. In duality $\left\langle C^{*}(G), B(G)\right\rangle$, for $f$ in $L^{1}(G)$ and $\phi$ in $B(G)$ the action of $\phi$ on $f$ is given by $\langle\phi, f\rangle=\int_{G} f(t) \phi(t) d t[25$, p. 192]. As proved in [25, Proposition 2.16], the space $B(G)$ with the pointwise multiplication and the dual norm

$$
\|\phi\|=\sup \left\{\left|\int_{G} f(t) \phi(t) d t\right|: f \in L^{1}(G),\|f\| \leq 1\right\}
$$

is a commutative Banach algebra, called the Fourier-Stieltjes algebra of $G$.

Let $A(G)$ denote the linear subspace of $C_{0}(G)$ consisting of all functions of the form $h * \tilde{f}$, where the functions $f$ and $h$ are in $L^{2}(G)$ and $\tilde{k}(s)=\overline{k\left(s^{-1}\right)}$. Let also $V N(G)$ be the von Neumann algebra in $B\left(L^{2}(G)\right)$ generated by the space $\left\{\rho(f): f \in C_{00}(G)\right\}$, where $C_{00}(G)$ is the subspace of $C_{0}(G)$ consisting of functions with compact supports. Then, each $\phi=h * \tilde{f}$ in $A(G)$ can be regarded as an ultraweakly continuous linear functional on $V N(G)$ defined by

$$
\phi(T)=\langle T(h), f\rangle \quad(T \in V N(G)) .
$$

P. Eymard proved that each ultraweakly continuous linear functionals on $V N(G)$ is of this form [25, Theorem 3.10]. Furthermore, $A(G)$ is the closed linear span of $P(G) \cap C_{00}(G)$ in $B(G)$. In particular, $A(G)$ is a closed ideal of $B(G)$, called 
the Fourier algebra of $G$. Also, the norm of an element $\phi$ in $A(G) \subseteq B(G)$ is the same as the norm of $\phi$ as a linear functional on $V N(G)$. As is well known, when $G$ is a locally compact abelian group, $A(G) \simeq L^{1}(\widehat{G}), B(G) \simeq M(\widehat{G})$ and $V N(G) \simeq L^{\infty}(\widehat{G})$. Here the sign $\simeq$ stands for "isometrically isomorphic" and $\widehat{G}$ is the dual group of the group $G$, see [25] for details. Also, when $G$ is compact, $A(G)=B(G)$.

Let $A$ be an arbitrary Banach algebra and $A_{1}$ be its closed unit ball. For $f$ in $A^{*}$, let $H(f)=\left\{a f: a \in A_{1}\right\}$. The functional $f$ is said to be "weakly almost periodic on $A$ " (resp. almost periodic on $A$ ) if the set $H(f)$, which is a convex bounded subset of $A^{*}$, is relatively weakly compact (resp. relatively compact). By $w a p(A)$ (resp. $a p(A)$ ) we denote the linear subspace of $A^{*}$ consisting of all weakly almost periodic (resp. almost periodic) functionals on $A$. The space $\operatorname{wap}(A)$ is a closed subspace of $A^{*}$ and the equality $\operatorname{wap}(A)=$ $A^{*}$ is equivalent to the fact $A$ is Arens regular, see for instance the paper [70]. We recall that on the second dual $A^{* *}$ of any Banach algebra $A$ there exist two algebra multiplications extending that of $A$ and that $A$ is said to be Arens regular if these multiplications coincide. Details of the construction of the multiplications can be found in many places including the book [5], the pioneering paper $[2,14]$ and the survey article [23]. See also the papers [29] and [70] for a functional analytic approach to this problem.

Next we recall definitions of some geometric properties we shall use in the subsequent sections. Let $X$ be a Banach space.

Radon-Nikodym property $(R N P)$. There exist several equivalent formulations of this property; here we shall adapt the following geometric definition. The Banach space $X$ is said to have the RNP if each closed convex subset $D$ of $X$ is dentable, i.e., for any $\varepsilon>0$ there exists an $x$ in $D$ such that $x \notin$ $\overline{\mathrm{Co}}\left(D \backslash B_{\varepsilon}(x)\right)$, where $B_{\varepsilon}(x)=\{y \in X:\|x-y\|<\varepsilon\}$. In this paper we shall mostly use the RNP on dual spaces through the following result: The space $X^{*}$ has the RNP if and only if every separable subspace of $X$ has separable dual [6, Corollary 4.1.7]. For more information on this notion we refer the reader to the books of Bourgin [6] and Diestel-Uhl [21].

Dunford-Pettis property (DPP). This notion, which has been introduced by $\mathrm{A}$. Grothendieck in [37], also has several equivalent formulations. Here we shall adapt the following one: The Banach space $X$ is said to have the DPP if, for any Banach space $Y$, every weakly compact linear operator $u: X \rightarrow Y$ sends weakly Cauchy sequences into norm convergent sequences. For more information on this property we refer the reader to Grothendieck's seminal paper [37] and to Diestel's survey paper [19].

Schur property. It is well known that in $l^{1}$ weakly convergent sequences are norm convergent. Any Banach space which shares this property with $l^{1}$ is said to have the Schur property. We shall use this property mostly in the following equivalent form: A Banach space $X$ has the Schur property if and only if every weakly compact subsets of $X$ are norm compact.

Flatness. Let $S=\{x \in X:\|x\|=1\}$ be the unit sphere of the Banach space $X$. The space $X$ is said to be flat if there exists a continuous function $g:[0,1] \rightarrow S$ with $g(1)=-g(0)$ and so that the length of the curve $g(t) \quad(0 \leq t \leq 1)$ is 2 , 
see [7, p. 18]. We are interested in this property only because of the fact that the flat Banach spaces do not have the RNP, see $[41,66]$ and references there.

Atomic von Neumann algebra. Let $M$ be a von Neumann algebra and $M_{*}$ be its predual. The algebra $M$ is said to be atomic if every nonzero projection in $M$ majorizes a nonminimal projection, see [62, p. 155].

Strongly finite von Neumann algebra. Again let $M$ be a von Neumann algebra. The algebra $M$ is said to be strongly finite if, for each $\phi$ in $M_{*}$, the set $\left\{v^{*} \phi v: v \in M^{u}\right\}$ is relatively norm compact. Here $M^{u}$ denotes the group of unitary elements of $M$, and $a \phi b$ is the element of $M_{*}$ defined by $\langle a \phi b, c\rangle=$ $\langle\phi, a c b\rangle$, for $a, b, c$ in $M$, see [35].

Direct sum of $C^{*}$-algebras. Let $\left(A_{\alpha}\right)_{\alpha \in I}$ be a family of $C^{*}$-algebras. By $\left(\sum_{\alpha} \oplus A_{\alpha}\right)_{\infty}$ we denote the $C^{*}$-algebra of all bounded families $a=\left(a_{\alpha}\right)_{\alpha \in I}$, with $a_{\alpha} \in A_{\alpha}$, equipped with the norm $\|a\|=\sup _{\alpha}\left\|a_{\alpha}\right\|$, the coordinatewise multiplication and involution. By $\left(\sum_{\alpha} \oplus A_{\alpha}\right)_{0}$ we denote the $C^{*}$-subalgebra of $\left(\sum \bigoplus A_{\alpha}\right)_{\infty}$ consisting of the families $a=\left(a_{\alpha}\right)$ which are null at infinity, i.e., $\forall \varepsilon>0,\left\|a_{\alpha}\right\|<\varepsilon$ for all but finitely many $\alpha$ in $I$.

Enveloping von Neumann algebra of a $C^{*}$-algebra. Let $A$ be a $C^{*}$-algebra. Every $C^{*}$-algebra being Arens regular, on the second dual $A^{* *}$ there exists a unique algebra multiplication extending that of $A$. The space $A^{* *}$ equipped with this unique multiplication is a $C^{*}$-algebra and is said to be the enveloping von Neumann algebra of $A$, and is denoted by $\tilde{A}$.

Now we recall some definitions about locally compact groups we shall need in later sections. Let $G$ be a locally compact group.

(a) The group $G$ is said to be an [AU]-group if the enveloping von Neumann algebra of the group $C^{*}$-algebra $C^{*}(G)$ is atomic. This is equivalent to saying that the von Neumann algebra generated by every continuous unitary representation of $G$ is atomic.

(b) The group $G$ is said to be an [AR]-group if the algebra $V N(G)$ is atomic. We clearly have the inclusions

$$
[\text { compact }] \subseteq[\mathrm{AU}] \subseteq[\mathrm{AR}] .
$$

Here [compact] denotes the collection of compact groups ... etc. These inclusions are proper, see [66].

(c) The group $G$ is called an [IN]-group if there is a compact neighbourhood of the identity $e$ of $G$ which is invariant under inner automorphisms.

(d) The group $G$ is called a [SIN]-group if there exists a base for the neighborhood system of $e$ consisting of compact sets invariant under inner automorphisms. Or, equivalently, the left and right uniformities of $G$ are the same. Obviously we have

$$
[\text { compact }] \subseteq[\mathrm{SIN}] \subseteq[\mathrm{IN}]
$$

and these inclusions are proper, see [53].

\section{3. $C^{*}$-Algebras SATISFYING The EQUALity $a p(A)=A^{*}$}

Let $A$ be a $C^{*}$-algebra and $A_{1}$ be its closed unit ball. We recall that, for $\phi$ in $A^{*}$ and $a$ in $A, a \phi: A \rightarrow \mathbb{C}$ is the functional defined by $\langle a \phi, b\rangle=\langle\phi, a b\rangle$ and that $\phi$ is said to be "almost periodic in $A$ " if the set $H(\phi)=\{a \phi: a \in$ 
$\left.A_{1}\right\}$ is relatively compact. This is equivalent to saying that the bounded linear operator $T_{\phi}: A \rightarrow A^{*}$, defined by $T_{\phi}(a)=\phi a$, is compact. Since, for $a$ in $A$, $T_{\phi}^{*}(a)=\phi a$, where $\phi a$ is defined by $\langle\phi a, b\rangle=\langle\phi, b a\rangle$ and $T_{\phi}^{*}$ is the adjoint of $T_{\phi}$, this is also equivalent to the fact that the set $\left\{\phi a: a \in A_{1}\right\}$ is relatively norm compact. The collection $a p(A)$ of all almost periodic functionals on $A$ is a closed linear subspace of $A^{*}$.

A result of [24] shows that for a commutative $C^{*}$-algebra $A=C(K)$ the equality $A^{*}=a p(A)$ holds if and only if the compact Hausdorff space $K$ is dispersed, i.e., $K$ has no nonempty perfect subset. Among 16 characterizations of the dispersed compact Hausdorff spaces given in [55] we note the following: The compact space $K$ is dispersed if and only if the space $C(K)$ does not contain an isomorphic copy of $l^{1}$ if and only if the dual space of $C(K)$ has the RNP. Our aim in this section is to show that similar results hold for any $C^{*}$-algebra $A$ satisfying the equality $a p(A)=A^{*}$. However, we should note that as far as the equality $a p(A)=A^{*}$ is concerned the basic difference between commutative and noncommutative $C^{*}$-algebras seems to be the DPP. Any commutative $C^{*}$-algebra has this property [37] whereas it is quite exceptional that a noncommutative $C^{*}$-algebra has this property, see the papers [39, 12 and 13] for results about $C^{*}$-algebra having the DPP.

For the proof of the main result of this section we need some preliminary results with which we now proceed. Let $(\Omega, \Sigma, \mu)$ be a probability space. We recall that the measure $\mu$ is purely atomic if and only if $\mu$ is of the form $\mu=\sum_{i=1}^{\infty} \lambda_{i} \delta_{t_{i}}$ with $\sum_{i=1}^{\infty} \lambda_{i}=1$ and $\delta_{t}$ is the Dirac measure at $t$, see [46, p. 31].

Lemma 3.1. The natural injection $i: L^{\infty}(\mu) \rightarrow L^{1}(\mu)$ is compact if and only if the measure $\mu$ is purely atomic.

Proof. First observe that the operator $i$ is always weakly compact for every finite measure $\mu$. Therefore $i$ is compact if and only if any uniformly bounded sequence $\left(\varphi_{n}\right)$ in $L^{\infty}(\mu)$ has a $\mu$-a.e. convergent subsequence. Now, assume $\mu$ is purely atomic and let $\left(\varphi_{n}\right)$ be a sequence in the unit ball of $L^{\infty}(\mu)$. Then a simple application of Cantor's Diagonal Method shows that $\left(\varphi_{n}\right)$ has a $\mu$-a.e. convergent subsequence so that $i$ is compact.

Conversely, assume that the operator $i$ is compact. Decompose $\mu$ as $\mu=$ $\lambda+\tilde{\mu}$, where $\lambda$ is an atomless measure, $\tilde{\mu}$ is a purely atomic measure and $\lambda \perp \tilde{\mu}$. Since $\lambda \perp \tilde{\mu}$, the natural injection $L^{\infty}(\lambda) \rightarrow L^{1}(\lambda)$ is also compact. But then the space $L^{1}(\lambda)$ is separable since $L^{\infty}(\lambda)$ is dense in $L^{1}(\lambda)$ and the unit ball of $L^{\infty}(\lambda)$, being relatively compact in $L^{1}(\lambda)$, is separable as a subset of $L^{1}(\lambda)$. Now, by Carathéodory's classification of atomless separable measure algebras [47, Theorem 5, p. 121], the Banach space $L^{1}(\lambda)$ is isometrically isomorphic to the Lebesgue space $L^{1}([0,1], m)$. It follows that the spaces $L^{\infty}(\lambda)$ and $L^{\infty}([0,1], m)$ are also isometrically isomorphic and the natural injection $L^{\infty}([0,1], m) \rightarrow L^{1}([0,1], m)$ is also compact. However this is not possible since, for instance, the uniformly bounded sequence $\varphi_{n}(x)=\sin (n \pi x)$ of $L^{\infty}([0,1], m)$ has no $m$-a.e. convergent subsequence. From this contradiction we conclude that the measure $\lambda$ is zero and $\mu=\tilde{\mu}$ is purely atomic.

Corollary 3.2. The set $\left\{f \cdot \varphi: \varphi \in L^{\infty}(\mu),\|\varphi\|_{\infty} \leq 1\right\}$ is relatively compact in $L^{1}(\mu)$ for each $f$ in $L^{1}(\mu)$ if and only if $\mu$ is purely atomic. 
Proof. If $\mu$ is purely atomic this is immediate to see. To see that the converse also holds, it is enough to take $f \equiv 1$ and apply the preceding lemma.

Another result we need for the proof of the main results of this section is the following lemma.

Lemma 3.3. Let $A$ be a $C^{*}$-algebra and $f$ an element of $A^{*}$. Then $f$ is almost periodic on $A$ if and only if the set $D=\left\{a f b: a, b \in A_{1}\right\}$ is relatively compact.

Proof. Assume $f$ is almost periodic on $A$. Then the sets $\left\{a f: a \in A_{1}\right\}$ and $\left\{f b: b \in A_{1}\right\}$ are relatively compact. Let $\varepsilon>0$ be an arbitrary number. Then there exist finitely many elements $a_{1}, a_{2}, \ldots, a_{n}$ in $A_{1}$ such that

$$
\left\{a f: a \in A_{1}\right\} \subseteq\left\{a_{1} f, \ldots, a_{n} f\right\}+\varepsilon A_{1}^{*} \text {. }
$$

Hence

$$
D=\left\{a f b: a, b \in A_{1}\right\} \subseteq \bigcup_{i=1}^{n}\left\{a_{i} f b: b \in A_{1}\right\}+\varepsilon A_{1}^{*},
$$

and the set $D$ is relatively compact by Lemma 2 , which is also valid for the norm topology, of [20, p. 227].

Conversely, assume that the set $D$ is relatively compact. Then since $A$ is Arens regular and has a bounded approximate identity $\left(e_{\alpha}\right)_{\alpha \in I}, f$ is of the form $a g$ for some $a$ in $A$ and $g$ in $A^{*}$, see [69, Corollary 3.2], for instance. Hence

$$
\left\|e_{\alpha} f-f\right\|=\left\|\left(a e_{\alpha}\right) g-a g\right\| \leq\|g\|\left\|a e_{\alpha}-a\right\| \rightarrow 0 .
$$

Now let $\varepsilon>0$ be arbitrary and choose an $\alpha$ in $I$ such that $\left\|e_{\alpha} f-f\right\|<\varepsilon$. Then,

$$
\left\{f b: b \in A_{1}\right\} \subseteq\left\{e_{\alpha} f b: b \in A_{1}\right\}+\varepsilon A_{1}^{*} \subseteq D+\varepsilon A_{1}^{*}
$$

so that again by $\left[20\right.$, Lemma 2, p. 227] the set $\left\{f b: b \in A_{1}\right\}$ is relatively compact, i.e., $f$ is almost periodic on $A$.

The following theorem is one of the main results of this section.

Theorem 3.4. Let $M$ be a von Neumann algebra and $M_{*}$ be its predual. Then the following assertions are equivalent.

(a) Each $\phi$ in $M_{*}$ is almost periodic on $M$.

(b) $M$ is the direct summand of finite dimensional $C^{*}$-algebras.

(c) On the unit ball of $M$ the multiplication is jointly weak*-continuous.

(d) The algebra $M$ is finite and atomic.

(e) The algebra $M$ is finite and $M_{*}$ is not flat.

(f) The algebra $M$ is finite and $M_{*}$ has the RNP.

(g) The space $M_{*}$ has the Schur property.

Proof. (a) $\Rightarrow($ b) . Assume that (a) holds. Then, by Lemma 3.3 above, the von Neumann algebra $M$ is strongly finite. Therefore, by Theorem 5.4 of [35], $M$ is of the form $Z \oplus\left(\sum \oplus A_{\alpha}\right)_{\infty}$. Here each $A_{\alpha}$ is a finite dimensional $C^{*}$ algebra and $Z$ is an abelian von Neumann algebra. Consequently, $Z$ is of the form $Z=\left(\sum_{\beta} \oplus L^{\infty}\left(\Omega_{\beta}, \mu_{\beta}\right)\right)_{\infty}$, where each $\left(\Omega_{\beta}, \mu_{\beta}\right)$ is a probability space [60, p. 46]. By Corollary 3.2 above, each $\mu_{\beta}$ is purely atomic. Hence $Z=l^{\infty}(\Gamma)$ for some set $\Gamma$. Since $l^{\infty}(\Gamma)$ is also a direct summand of a family of one dimensional $C^{*}$-algebras, we see that $M$ is a direct summand of the finite dimensional $C^{*}$-algebras. 
(b) $\Rightarrow$ (c). Assume $M$ is of the form $M=\left(\sum_{\alpha} \bigoplus A_{\alpha}\right)_{\infty}$, where each $A_{\alpha}$ is a finite dimensional $C^{*}$-algebra. Let $\tau$ be the topology of pointwise convergence on $I$, i.e., the product topology on $\prod_{\alpha} A_{\alpha}$. Then, the space $\left(M_{1}\right.$, weak $\left.{ }^{*}\right)$ is compact and the space $\left(M_{1}, \tau\right)$ is Hausdorff. Moreover the weak*-topology is stronger than $\tau$. Hence these two topologies agree on $M_{1}$. Since the multiplication on $M_{1}$ is clearly jointly continuous for the topology $\tau$, it also is jointly continuous in the weak*-topology.

(c) $\Rightarrow(\mathrm{a})$. Assume the multiplication is jointly continuous on $M_{1}$ in the weak*-topology. Then the space $\left(M_{1}\right.$, weak $\left.{ }^{*}\right)$ is a compact topological semigroup. Hence $C\left(M_{1}\right)=A P\left(M_{1}\right)$, where $A P\left(M_{1}\right)$ denotes the space of continuous almost periodic functions on $\left(M_{1}\right.$, weak $\left.{ }^{*}\right)$, see [18]. It follows that each $\phi$ in $M_{*}$ is almost periodic.

The equivalences (d) $\Leftrightarrow$ (e) $\Leftrightarrow$ (f) hold since, by Theorem 4.2 of [66], $M$ is atomic if and only if $M_{*}$ is not flat if and only if $M_{*}$ has the RNP. Also the equivalence $(\mathrm{d}) \Leftrightarrow(\mathrm{g})$ is established in [59, Theorems 1 and 2] and [39, Theorem 3].

(a) $\Rightarrow(\mathrm{d})$. Assume (a) holds. Then, by Lemma 3.3 above, $M$ is strongly finite, so finite [73]. Also, by (b), $M$ is of the form $M=\left(\sum_{\alpha} \bigoplus A_{\alpha}\right)_{\infty}$, where each $A_{\alpha}$ is a finite dimension $C^{*}$-algebra. Let $A=\left(\sum_{\alpha} \bigoplus A_{\alpha}\right)_{0}$. Then $M$ is the enveloping von Neumann algebra of $A$, and $M$ is atomic [62, p. 157].

$(\mathrm{g}) \Rightarrow(\mathrm{a})$. Assume $(\mathrm{g})$ holds. Then for each $\phi$ in $M_{*}$, by Arens regularity of $M$, the set $\left\{a \phi: a \in M_{1}\right\}$ is relatively weakly compact, so relatively norm compact since the space $M_{*}$ has the Schur property.

The next lemma, which is needed for the proof of Theorem 3.6 below, is actually a corollary of Lemma 3.3 above.

Lemma 3.5. Let $A$ be a $C^{*}$-algebra, $\widetilde{A}$ its enveloping von Neumann algebra and $f$ an element of $A^{*}$. Then $f$ is almost periodic on $A$ if and only if the set $H=\left\{a f b: a, b \in \widetilde{A_{1}}\right\}$ is relatively compact.

Proof. Assume $f$ is almost periodic on $A$. Then the set $D=\left\{a f b: a, b \in A_{1}\right\}$ is relatively compact by Lemma 3.3 above. Hence $\bar{D}$, the norm closure of $D$, is a norm compact (so weak*-closed) subset of $A^{*}$. Let $\tilde{a}, \tilde{b}$ be two elements in $\tilde{A}_{1}$. By the Goldstine lemma, there exist nets $\left(a_{\alpha}\right)$ and $\left(b_{\beta}\right)$ in $A_{1}$ such that $a_{\alpha} \rightarrow \tilde{a}$ and $b_{\beta} \rightarrow \tilde{b}$ in the weak*-topology of $\tilde{A}$. Now it is immediate to see that, in the weak*-topology of $A^{*}$,

$$
a_{\alpha} f b_{\beta} \rightarrow \tilde{a} f b_{\beta} \text { and } \tilde{a} f b_{\beta} \rightarrow \tilde{a} f \tilde{b}
$$

so that the set $H$ is contained in $\bar{D}$, and it is relatively compact. The converse is immediate.

In the next theorem we give several characterizations of $C^{*}$-algebras satisfying the equality $A^{*}=a p(A)$. This answers a question raised by Professor John Duncan during the regional conference of the American Mathematical Society held in Fayetteville (Arkansas), March 1990. For the proof of this theorem, we use the following result: The dual space $X^{*}$ of a Banach space has the Schur property if and only if $X$ has the DPP and $X$ does not contain a copy of $l^{1}$, see Theorem 3 and the remark following it in [19, p. 23].

Theorem 3.6. Let $A$ be a $C^{*}$-algebra. Then the following assertions are equivalent. 
(a) $\operatorname{ap}(A)=A^{*}$.

(b) The space $A^{*}$ is an $l^{1}$-sum of finite dimensional Banach spaces.

(c) The space $A^{*}$ has the Schur property.

(d) The space $A^{*}$ has the RNP and the DPP.

(e) The space $A^{*}$ is not flat and has the DPP.

(f) The algebra $A$ is scattered and has the DPP.

(g) The algebra $A$ does not contain a copy of $l^{1}$ and has the DPP.

(h) Every bounded linear operator $T: A \rightarrow A^{*}$ is compact.

(i) Every irreducible representation of $A$ is finite dimensional and $A^{*}$ has the $R N P$.

Proof. Theorem 3.4 above applied to the von Neumann algebra $A^{* *}$ shows that the implications (a) $\Rightarrow$ (b) and (c) $\Rightarrow$ (a) hold. By [28, Corollary VII.10], $A^{*}$ has the RNP if and only if $A$ does not contain an isomorphic copy of $l^{1}$. By [66, Theorem 3.5], $A^{*}$ has the RNP if and only if $A^{*}$ is not flat. We also know that $A$ has the DPP if $A^{*}$ has it [37, Corollary of Proposition 1, p. 136]. These facts combined show that the implications (d) $\Leftrightarrow(\mathrm{e}) \Rightarrow(\mathrm{f}) \Leftrightarrow(\mathrm{g})$ hold. The implication (f) $\Rightarrow$ (e) holds by Theorem 7 of [4]. The equivalence $(\mathrm{g}) \Leftrightarrow$ (c) follows from the note preceding this theorem. To prove the implication $(\mathrm{g}) \Rightarrow(\mathrm{h})$, assume that $(\mathrm{g})$ holds. Then the space $A^{*}$ has the Schur property. On the other hand, as an immediate consequence of the Grothendieck-PisierHaagerup inequality [38], every bounded linear operator $T: A \rightarrow A^{*}$ is weakly compact, so compact. The implication $(\mathrm{h}) \Rightarrow(\mathrm{a})$ is trivial since to say that a functional $f$ in $A^{*}$ is almost periodic is equivalent to saying that the bounded linear operator $T_{f}: A \rightarrow A^{*}$ defined by $T_{f}(a)=a f$ is compact. To prove that $(\mathrm{b}) \Rightarrow(\mathrm{c})$ assume that $A^{*}=\left(\sum_{\alpha} \bigoplus X_{\alpha}\right)_{1}$, where each $X_{\alpha}$ is a finite dimensional Banach space. Then by [17, Lemma, p. 31] $A^{*}$ has the DPP. Now if we put $B=\left(\sum_{\alpha} \bigoplus X_{\alpha}\right)_{0}$, then $B^{*}=A^{*}$. It follows that $B$ also has the DPP [17, Corollary of Proposition 1, p. 136]. Since $B$ does not contain an isomorphic copy of $l^{1}$, again by the note preceding the statement of this theorem, we conclude that $A^{*}$ has the Schur property. Finally, the equivalence (f) $\Leftrightarrow$ (i) follows from [39, Theorem 1].

Remark 3.7. (a) In view of the implication (a) $\Rightarrow$ (b) and its proof it seems likely that any $C^{*}$-algebra $A$ satisfying the equality $a p(A)=A^{*}$ is of the form

$$
A=C_{0}(S) \bigoplus\left(\sum_{\alpha} \bigoplus A_{\alpha}\right)_{0},
$$

where each $A_{\alpha}$ is a finite dimensional $C^{*}$-algebra and $S$ is a dispersed locally compact Hausdorff space determined (within a homeomorphism) by the algebra $A$ (note that $C_{0}(S)$ may be the center of $A$ ). However as the dual $A^{*}$ of a $C^{*}$-algebra does not determine (within an isometric isomorphism) uniquely its predual, from the implication (a) $\Rightarrow$ (b) we cannot conclude that $A$ is of this form.

(b) In contrast with commutative $C^{*}$-algebra, where, for $A=C(K), A^{*}=$ $a p(A)$ if and only if $K$ is dispersed [24], there exist scattered noncommutative $C^{*}$-algebras $A$ with $a p(A)=\{0\}$. Indeed, if $H$ is an infinite dimensional Hilbert space and $A=K(H)$ is the $C^{*}$-algebra of compact linear operators on $H$ then $A$ is scattered [10] and $a p(A)=\{0\}$ [24, Proposition 3.3]. As far as the equality $A^{*}=\operatorname{ap}(A)$ is concerned the basic difference between commutative and noncommutative $C^{*}$-algebras seems to be the DPP. It is well known that 
the algebra $K(H)$ lacks this property, see [39, Lemma 2] and also the following simple proposition.

Proposition 3.8. Let $A$ be a Banach algebra which is an ideal in its second dual. If $\operatorname{ap}(A)=\{0\}$ then $A$ cannot have the DPP.

Proof. Since $A$ is an ideal in its second dual (equipped with either Arens multiplication), for each element $a$ in $A$, the multiplication operator $\tau_{a}: A \rightarrow A$ defined by $\tau_{a}(x)=a x$ is weakly compact [23, Lemma 3, p. 318]. Should $A$ have the DPP, for any $b$ and $c$ in $A$, with $a=b c$, the operator $\tau_{a}=\tau_{b} \circ \tau_{c}$ would be compact. But then, for each $f$ in $A^{*}$, the functional $g=a f$ would be almost periodic, contradicting the hypothesis that $a p(A)=\{0\}$. Therefore $A$ does not have the DPP.

While passing we remark here that if $X$ is a reflexive Banach space with the approximation property then the algebra $A=K(X)$ is an ideal in its second dual $B(X)$ and since $a p(A)=\{0\}$ [24, Proposition 2.3] for no $u$ in $K(X)$, the multiplication operator $\tau_{u}: K(X) \rightarrow K(X), \tau_{u}(v)=u \circ v$, is compact.

\section{SOME GEOMETRIC PROPERTIES ON FOURIER AND FOURIER-STIELTJES ALGEBRAS}

In this section we apply the results obtained in the preceding section to the Fourier algebra $A(G)$ and the Fourier-Stieltjes algebra $B(G)$ associated to a locally compact group $G$.

For the proof of the main results of this section we need the following lemma, which is of independent interest. This lemma indicates a practical method of proving that a dual $C^{*}$-algebra has the DPP, see especially the equivalence (c) $\Leftrightarrow(\mathrm{d})$ and Lemma 8.6 below.

Lemma 4.1. Let $H$ be a Hilbert space and let $A$ be a $C^{*}$-subalgebra of $K(H)$. Then the following assertions are equivalent.

(a) $\operatorname{ap}(A)=A^{*}$.

(b) The space $A^{*}$ has the Schur property.

(c) The space $A$ has the DPP.

(d) For each $a$ in $A$, the multiplication operator $\tau_{a}: A \rightarrow A$ defined by $\tau a(x)=x a$ is compact.

(e) $A$ is a $c_{0}$-sum of a family of finite dimensional $C^{*}$-algebras.

Proof. Since the algebra $K(H)$ is scattered [10], the algebra $A$ does not contain a copy of $l^{1}$. Therefore the equivalences $(a) \Leftrightarrow(b) \Leftrightarrow(c)$ hold by Theorem 3.6 of the preceding section. To prove the implication (c) $\Rightarrow(\mathrm{d})$, assume that $A$ has the DPP. Let $a$ be an element in $A$. Then by the Cohen-Hewitt Factorization Theorem [42, 32.22], $a=b c$ for some $b$ and $c$ in $A$. On the other hand, since $K(H)$ is an ideal in its second dual $B(H)$, so is $A$ and consequently the multiplication operators $\tau_{b}$ and $\tau_{c}$ are weakly compact. Hence, since the algebra $A$ has the DPP, the operator $\tau_{a}=\tau_{b} \circ \tau_{c}$ is compact. [Of course, for the same reason, the operator $x \mapsto a x$ is also compact.] The equivalence $(d) \Leftrightarrow(e)$ is proved in [1, Corollary 2.6]. The implication $(e) \Rightarrow(a)$ is trivial.

We recall that a locally compact group $G$ is said to be a Moore group, which is denoted by $G \in$ [MOORE], if each irreducible continuous unitary representation of $G$ is finite dimensional, see [53]. Clearly all compact groups and all 
abelian groups are Moore groups. Let $W^{*}(G)=C^{*}(G)^{* *}$. Then we have the following result.

Theorem 4.2. Let $G$ be a locally compact group. Then the following assertions are equivalent.

(a) $G \in[M O O R E]$.

(b) The space $B(G)$ has the DPP.

(c) The space $C^{*}(G)$ has the DPP.

(d) The von Neumann algebra $W^{*}(G)$ is finite.

(e) The von Neumann algebra $W^{*}(G)$ is finite and of type 1.

(f) The von Neumann algebra $V N(G)$ is finite and type 1.

Moreover when $G$ is discrete, each of the above assertions is equivalent to

(g) $G$ has an abelian subgroup of finite index.

Proof. Since irreducible continuous unitary representations of $G$ correspond to irreducible representation of the $C^{*}$-algebra $C^{*}(G)[22,13.9]$, the equivalences (a) $\Leftrightarrow(\mathrm{b}) \Leftrightarrow$ (c) $\Leftrightarrow$ (d) $\Leftrightarrow$ (e) are a direct consequence of [39, Theorem 1] and the theorem in [12]. Also, the equivalence $(a) \Leftrightarrow$ (f) follows from [44, Theorem 3]. Finally, the equivalence $(\mathrm{a}) \Leftrightarrow(\mathrm{g})$ follows from [67], See also [45 and 61].

The first main result of this section is the following theorem.

Theorem 4.3. Let $G$ be a locally compact group. Then the following assertions are equivalent.

(a) $G$ is compact.

(b) Each $\phi$ in $A(G)$ is almost periodic on the algebra $V N(G)$.

(c) On the unit ball of $V N(G)$ the multiplication is jointly continuous in the $\sigma$-topology.

(d) The algebra $V N(G)$ is the direct summand of finite dimensional $C^{*}$ algebras.

(e) $G \in[I N] \cap[A R]$.

(f) $G \in[I N]$ and the space $A(G)$ is not flat.

(g) $G \in[I N]$ and the space $A(G)$ has the RNP.

(h) The space $A(G)$ has the Schur property.

Proof. The equivalences (b) $\Leftrightarrow$ (c) $\Leftrightarrow$ (d) $\Leftrightarrow$ (h) are direct consequences of Theorem 3.4 above. The equivalences (e) $\Leftrightarrow$ (f) $\Leftrightarrow$ (g) follow from [66, Theorem 4.2]. The implication $(\mathrm{a}) \Rightarrow(\mathrm{h})$ follows from Theorem 4.2 and Lemma 4.1 above since every compact group is in [MOORE]. To prove the implication (a) $\Rightarrow(\mathrm{g})$, it is enough to prove that the space $A(G)$ has the RNP, which is an immediate consequence of [66, Corollary 3.7] since, for $G$ compact, $C^{*}(G)$ is a dual $C^{*}$-algebra (see also [34, Theorem B1]). To prove the implication (c) $\Rightarrow(\mathrm{a})$, assume that (c) holds but $G$ is not compact. Let $\left(x_{\alpha}\right)$ be a net in $G$ that converges to "infinity." Let $L_{x_{\alpha}}: L^{2}(G) \rightarrow L^{2}(G)$ be the operator defined by $L_{x_{\alpha}}(h)=h\left(x_{\alpha}^{-1} y\right)$. Then, if necessary passing to a subnet, we can assume that the net $\left(L_{x_{\alpha}}\right)$ converges in the $\sigma$-topology of $V N(G)$ to some element $T$ of $V N(G)$. Now, since $A(G) \subseteq C_{0}(G)$, for each $\phi$ in $A(G)$,

$$
\left\langle\phi, L_{x_{\alpha}}\right\rangle=\phi\left(x_{\alpha}\right) \rightarrow 0 \text {. }
$$

Hence $\langle\phi, T\rangle=0$ for all $\phi$ in $A(G)$, and $T=0$. It follows that zero is in the set $\widetilde{G}=\left\{\overline{L_{x}: x \in G}\right\}^{\sigma}$. Since the mapping $x \mapsto L_{x}$ is a homeomorphism from $G$ into $\widetilde{G}$, the set $\widetilde{G}$ equipped with the $\sigma$-topology has jointly continuous 
multiplication. But this is not possible since otherwise the space $(\widetilde{G}, \sigma)$ would be a compact group, which is impossible.

To complete the proof it remains to show that (e) $\Rightarrow(\mathrm{a})$. Assume $G$ is in $[\mathrm{IN}] \cap[\mathrm{AR}]$. Then, since $G$ is in [IN], there exists a compact normal subset $K$ of $G$ such that $G / K$ is a [SIN]-group [53, p. 718]. On the other hand, since $G$ is in [AR], the space $A(G)$ has the RNP [66, Theorem 4.1]. As $A(G / K)$ is isometrically isomorphic to the closed subalgebra of $A(G)$ consisting of functions constant on cosets of $K$ [25], $A(G / K)$ also has the RNP. Hence by Theorem 3.4 above, the multiplication of $V N(G / K)$ is jointly continuous on its unit ball for the $\sigma$-topology. Hence, by the proof of the implication (c) $\Rightarrow$ (a), $G / K$ is compact. The groups $K$ and $G / K$ being both compact, by [42, 5.25], $G$ itself is compact.

Remark 4.4. (a) The equivalence (a) $\Leftrightarrow$ (e) improves Theorem 4.7 of K. Taylor [66].

(b) The condition that $G$ is in [IN] cannot be dropped from conditions of assertions (e), (f) or (g). Indeed, if $G$ is the " $a x+b$-group" then $G$ not being unimodular, is not an [IN]-group [53]. However $G$ is in [AR] by [4].

The next theorem is a direct consequence of Theorem 3.6, Lemma 4.1, Theorem 4.2 and the fact that when $G$ is compact $A(G)=B(G)$.

Theorem 4.5. Let $G$ be a locally compact group. Then the following assertions are equivalent.

(a) $G$ is compact.

(b) The space $B(G)$ is an $l^{1}$-sum of finite dimensional Banach spaces.

(c) The space $B(G)$ has the Schur property.

(d) The space $B(G)$ has the RNP and the DPP.

(e) $G \in[M O O R E]$ and the space $B(G)$ has the $R N P$.

(f) The algebra $C^{*}(G)$ is scattered and has the DPP.

(g) The space $C^{*}(G)$ does not contain a copy of $l^{1}$ and has the DPP.

(h) Every bounded linear operator $T: C^{*}(G) \rightarrow B(G)$ is compact.

(i) The space $B(G)$ is not flat and has the DPP.

(j) $\operatorname{ap}\left(C^{*}(G)\right)=B(G)$.

Now let $P_{\rho}(G)$ be the closure of $P(G) \cap C_{00}(G)$ in the compact-open topology of $B(G)$ and $B_{\rho}(G)$ be the linear span of $P_{\rho}(G)$. Then $B_{\rho}(G)$ is a closed ideal of $B(G)$ containing $A(G)$ and $B_{\rho}(G)$ is precisely the dual of $C_{\rho}^{*}(G)$ [25, Propositions 2.1 and 2.16]. As known [36, p. 61], $B_{\rho}(G)=B(G)$ if and only if $G$ is amenable. We denote by $\widehat{G}_{r}$ the reduced dual of $G$, see [22, p. 357].

Theorem 4.6. Let $G$ be a locally compact group. Then the following assertions are equivalent.

(a) $G$ is compact.

(b) The space $B(G)$ is an $l^{1}$-sum of finite dimensional Banach spaces.

(c) The space $B_{\rho}(G)$ has the Schur property.

(d) The space $B_{\rho}(G)$ has the RNP and the DPP.

(e) Each $\sigma$ in $\widehat{G}_{r}$ is finite dimensional and $B_{\rho}(G)$ has the RNP.

(f) The algebra $C_{\rho}^{*}(G)$ is scattered and has the DPP.

(g) The space $C_{\rho}^{*}(G)$ does not contain a copy of $l^{1}$ and has the DPP.

(h) Every bounded linear operator $T: C_{\rho}^{*}(G) \rightarrow B_{\rho}(G)$ is compact. 
(i) $B_{\rho}(G)$ is not flat and has the DPP.

(j) $\operatorname{ap}\left(C_{\rho}^{*}(G)\right)=B_{\rho}(G)$.

Proof. The equivalences

$$
\text { (b) } \Leftrightarrow(\mathrm{c}) \Leftrightarrow(\mathrm{d}) \Leftrightarrow(\mathrm{e}) \Leftrightarrow(\mathrm{f}) \Leftrightarrow(\mathrm{g}) \Leftrightarrow(\mathrm{h}) \Leftrightarrow(\mathrm{i}) \Leftrightarrow(\mathrm{j})
$$

follow from Theorem 3.5. Also, the implication (a) $\Rightarrow$ (b) follows from Theorem 4.5 and the fact that $B_{\rho}(G)=B(G)$ when $G$ is compact. Finally, if (c) holds then $A(G)$, being a closed subspace of $B_{\rho}(G)$, also has the Schur property. Hence by Theorem 4.3, $G$ is compact.

As shown in [66], a locally compact group $G$ is an [AU]-group (resp. [AR]group) if and only if the space $B(G)$ (resp. $A(G)$ ) has the RNP. The RNP being a remarkably stable property one can exploit these equivalences to obtain short and functional analytic proof of some properties of [AU]- and [AR]-groups. Below we present some results in this direction, compare with [66, Theorem 4.4].

Proposition 4.7. Let $G$ and $G_{1}$ be two locally compact groups with $G \in[\mathrm{AU}]$. Then

(a) If there exists a continuous homomorphism $\sigma: G \rightarrow G_{1}$ with $\sigma(G)$ dense in $G_{1}$ then $G_{1} \in[\mathrm{AU}]$ too.

(b) If $H$ is a closed normal subgroup of $G$ then $G / H$ is also an [AU]-group. Proof. (a) Let $J: B\left(G_{1}\right) \rightarrow B(G)$ be the mapping defined by $j(u)=u \circ \sigma$. Then, by Theorem 2.20 of Eymard [25], $j$ is a linear isometry so that we can identify $B\left(G_{1}\right)$ with a closed subspace of $B(G)$. Since the space $B(G)$ has the RNP, the space $B\left(G_{1}\right)$ also has this property. Hence $G_{1}$ is an [AU]-group.

(b) Let $\sigma: G \rightarrow G / H$ be the natural surjection. Then, by (a), $G / H$ is also an [AU]-group.

Remark 4.8. Let $G$ be an infinite discrete group and $G^{a}$ be its almost periodic compactification. The space $G^{a}$, being a compact group, is an [AU]-group whereas $G$, being infinite, is not an [AU]-group [66, Corollary 4.8]. This remark shows that an open dense subgroup of an [AU]-group need not be an [AU]-group.

In contrast with the preceding remark we have the following result.

Proposition 4.9. An open subgroup $G_{1}$ of an $[A R]$-group is an [AR]-group.

Proof. By proposition 3.21(1) of [25], the algebra $A\left(G_{1}\right)$ is isometrically isomorphic to a subalgebra of $A(G)$. As $A(G)$ has the RNP, the space $A\left(G_{1}\right)$ also has the same property so that $G_{1}$ is an [AU]-group.

Proposition 4.10. Let $G$ be an $[A R]$-group and $K$ a compact normal subgroup of $G$. Then the group $G / K$ is also an $[A R]$-group.

Proof. By Proposition 3.25 of Eymard [25], the space $A(G / K)$ is isometrically isomorphic to a subspace of $A(G)$. It follows that the space $A(G / K)$ has the $\mathrm{RNP}$ and $G / K$ is an [AU]-group.

The last result of this section is the proposition below about separability of the space $B(G)$ for the proof of which we need the following lemma.

Lemma 4.11. Let $A$ be a separable $C^{*}$-algebra. Then $A^{*}$ is separable if and only if $\operatorname{ext} S(A)$, the set of extreme points in the state space $S(A)$ of $A$, is norm separable. 
Proof. If $A^{*}$ is separable then the space $\operatorname{ext} S(A)$ is separable since a separable subspace of a separable metric space is separable. Conversely, assume ext $S(A)$ is separable. Then an argument similar to the one in [20, Theorem, p. 161] shows that $S(A)$ is contained in the norm closure $\overline{\mathrm{Co}}(\operatorname{ext} S(A))$ of the convex hull of ext $S(A)$. Since $A^{*}$ is the linear span of $S(A)$, it follows that $A^{*}$ is also norm separable.

Let $A$ be a $C^{*}$-algebra. For each $f \in \operatorname{ext} S(A)$, let $\pi_{f}$ be the representation of $A$ associated to $f$. For $f, g$ in ext $S(A)$, write $f \sim g$ if and only if $\pi_{f}$ and $\pi_{g}$ are unitarily equivalent. Then " $\sim$ " is an equivalence relation on $S(A)$. Let $\dot{f}$ be the equivalence class of $f \in \operatorname{ext} S(A)$. Form a new set $E(A)$ by taking one element $f$ in each $\dot{f}$. Then clearly, Card $E(A)=\operatorname{Card} \widehat{A}$, where $\widehat{A}$ denotes the spectrum of $A$.

Lemma 4.12. If $A$ is a $C^{*}$-algebra and $A^{*}$ is separable, then $E(A)$ is countable. Proof. Since the addition of unit has no effect on the representation of a $C^{*}$ algebra, we may assume that $A$ is unital. In this case, if $f, g \in E(A), f \neq g$, then $\|f-g\| \geq 2$ (see [22, p. 64]). Thus $E(A)$ equipped with the metric induced by the norm of $A^{*}$ is uniformly discrete. Hence $E(A)$ is countable.

Proposition 4.13. Let $G$ be a locally compact group. Then the following are equivalent.

(a) The space $B(G)$ is separable

(b) The subset ext $P_{1}(G)$ of $B(G)$ is separable, where $P_{1}(G)=\{\phi: \phi \in P(G)$ and $\phi(e)=1\}$.

(c) The space $C^{*}(G)$ is separable and does not contain a copy of $l^{1}$.

(d) $G$ is second countable and $\widehat{G}$ is countable.

In this case $G$ is unimodular and contains a compact open subgroup.

Proof. We first remark that the set $P_{1}(G)$ can be identified with the state space of the $C^{*}(G)$. Now the equivalence $(\mathrm{a}) \Leftrightarrow$ (b) follows from the Lemma 4.11. As for the equivalence $(\mathrm{a}) \Leftrightarrow(\mathrm{c})$, it is an immediate consequence of the fact that the dual space of a separable Banach space $X$ has the RNP if and only if $X^{*}$ is separable [6, Corollary 4.17].

(a) $\Rightarrow$ (d) If $B(G)$ is separable, then $\sigma(B(G))$, the spectrum of $B(G)$, is compact and metrizable, and hence second countable. Consequently $G$, which is homeomorphic to a subspace of $\sigma(B(G))$, is second countable. Also, by Lemma 4.12 (or [66, Theorem 4.5]), $\widehat{G}$ is countable.

(d) $\Rightarrow$ (a) If $G$ is separable and $\widehat{G}$ is second countable, by Theorem 4.5 in [66], $B(G)$ has RNP. Since $C^{*}(G)$ is separable, $B(G)$ must also be separable [6, Corollary 4.17].

The final statement follows from [73, Theorem 6.2].

Remarks and Questions 4.14 . Let $G$ be a locally compact group.

(a) If $G$ is compact then the space $A(G)$ has the DPP by Theorem 4.3. On the reverse direction, if $A(G)$ has the DPP then the algebra $V N(G)$ is finite [39, Lemma 4] so that $G$ is a [SIN]-group [22, 13.10.5]. We do not know for which groups exactly the space $A(G)$ has the DPP.

(b) If $G$ is compact then the space $A(G)$ is a dual space. But the converse is not true. In fact, if $G$ is second countable (or more generally, when $A(G)$ is weakly compactly generated), then $A(G)$ is a dual space if and only if $G$ is 
an [AR]-group (see [66, Theorem 4.1] and [11]). As shown in [4], the " $a x+b$ "group is a second countable noncompact [AR]-group. Also if $G \in[\mathrm{AR}]$, then $A(G)$ is dual [66, Theorem 3.5]. However, we do not know if the converse is true without countability assumption.

(c) When is $A(G)$ weakly compactly generated (or equivalently, the von Neumann algebra is $\sigma$-finite [11, Lemma])? This is the case when $G$ is second countable.

(d) We do not know if there exists a locally compact group $G$ such that the space $B_{\rho}(G)$ has the RNP (resp. DPP) and the space $B(G)$ lacks it.

\section{The CASE OF THE GROUP Algebra AND THE MEASURe Algebra}

In this section we apply the results established in $\S 3$ to the group algebra $L^{1}(G)$ and the measure algebra $M(G)$. Note that when $G$ is abelian we have $A(G)=L^{1}(\widehat{G})$ and $B(G)=M(\widehat{G})$, where $\widehat{G}$ is the dual group of $G$. We also note that the spaces $M_{1}(G), L^{\infty}(G), L^{1}(G)$, and $C_{0}(G)$ have all the DPP [37].

The next theorem is a direct application of Theorem 3.4 and the fact that the space $C_{0}(G)$ does not contain a copy of $l^{1}$ if and only if $G$ is dispersed [55]. We also note that a locally compact group is dispersed if and only if $G$ is discrete.

Theorem 5.1. Let $G$ be any locally compact group. Then the following are equivalent.

(a) The group $G$ is discrete.

(b) Each $f$ in $L^{1}(G)$ is almost periodic on $L^{\infty}(G)$.

(c) The space $L^{1}(G)$ has the RNP.

(d) The space $L^{1}(G)$ is not flat.

(e) The space $L^{1}(G)$ has the Schur property.

(f) On the unit ball of $L^{\infty}(G)$ the multiplication is jointly continuous in the weak*-topology.

(g) The space $M(G)$ has the RNP.

(h) The space $M(G)$ has the Schur property.

(i) The space $C_{0}(G)$ does not contain a copy of $l^{1}$.

(j) The space $C_{0}(G)$ is scattered.

(k) Every bounded linear operator $T: C_{0}(G) \rightarrow M(G)$ is compact.

We shall denote by $A P(G)$ and $W A P(G)$ the spaces of continuous almost periodic and weakly almost periodic functions on $G$, respectively.

Corollary 5.2. Let $G$ be a locally compact group. Then the space $A P(G)^{*}$ has the $R N P$ (or the Schur property) if and only if the space $A P(G)$ is finite dimensional.

Proof. As is well known $A P(G)=C\left(G^{a}\right)$, where $G^{a}$ is the almost periodic compactification of $G$. The space $G^{a}$ being a compact group, the conclusion follows from the preceding theorem.

At this point we remark that even if $G$ is infinite the space $A P(G)$ may be trivial i.e., consists of constant functions, see [9]. Compare this remark with Remark 3.7(b).

Corollary 5.3. Let $G$ be a noncompact locally compact group and $A$ be a $C^{*}$ subalgebra of $C(G)$ containing $C_{0}(G)$ and constant functions. Then $A$ has the $R N P$ (or the Schur property) if and only if $G$ is discrete and $A=C_{0}(G) \oplus \mathbb{C}$. 
Proof. The implication $(\Leftarrow)$ is clear. To prove the other implication, assume that $A^{*}$ has the RNP (or the Schur property). Then, by Theorem 5.1 above, $\operatorname{ap}(A)=A^{*}$. Each $\phi$ in $C_{0}(G)^{*}$ extends by the Hahn-Banach Theorem to a functional on $A$, which is almost periodic on $A$. It follows that each functional in $C_{0}(G)^{*}$ is almost periodic on $C_{0}(G)$, and $G$ discrete. Now let $\Delta$ be the spectrum of $A$ and, for $x$ in $G$, let $\hat{x}$ be the point evaluation at $x$. Then the mapping $x \mapsto \hat{x}$ is an embedding of $G$ onto a dense subset of $\Delta$. Let $E=\Delta \backslash \widehat{G}$, where $\widehat{G}$ is the image of $G$ under the preceding embedding. Then each $\theta$ in $E$ is an accumulation point of $E$, which contradicts the fact that $\Delta$ is dispersed (since $A^{*}$ has the RNP or the Schur property) unless $E$ is a one-point set, i.e., $\Delta$ is the one-point compactification of $G$. It follows that $A=C_{0}(G) \oplus \mathbb{C}$.

Corollary 5.4. Let $G$ be a locally compact group. Then the space $W A P(G)^{*}$ has the RNP (or the Schur property) if and only if $G$ is finite.

Proof. First assume $G$ is compact and the space $W A P(G)^{*}=C(G)^{*}$ has the RNP (or the Schur property). Then, by Theorem 5.1 above, $G$ is discrete, so finite. If $G$ is noncompact and the space $W A P(G)^{*}$ has the RNP (or the Schur property) then, the preceding corollary applied to $W A P(G)$ shows that $G$ is discrete and $W A P(G)=C_{0}(G) \oplus \mathbb{C}$. Hence $G$ is both minimally almost periodic and minimally weakly almost periodic, which is not possible if $G$ is infinite [8]. The converse is trivial.

\section{FINE LINE BETWEEN COMPACT GROUP AND [AU]-GROUPS}

The main result of this section (Theorem 6.4) draws a fine line between dual $C^{*}$-algebras and scattered $C^{*}$-algebras. Since the algebra $C^{*}(G)$ is dual (resp. scattered) if and only if $G$ is compact (Lemma 6.6 below) (resp. $G$ is an [AU]-group), the main result also draws a fine line between compact groups and [AU]-groups. Since the algebra $C^{*}(G)$ is dual (resp. scattered) if and only if the space $B(G)$ has the Schur property (resp. the RNP), the main result can also be interpreted as a result that draws a fine line between the Schur property and the RNP on $B(G)$. However this interpretation is not valid for an arbitrary $C^{*}$-algebra even if the $C^{*}$-algebra in question is commutative. Indeed, if $K$ is a nondiscrete dispersed compact Hausdorff space and $A=C(K)$ then $A^{*}$ has the Schur property as well as the RNP but $A$ is not a dual algebra. The main result characterizes the scattered $C^{*}$-algebras in terms of operators $\tau_{a}$, where $\tau_{a}$ is the multiplication operator on the given algebra $A$, i.e., $\tau_{a}(x)=a x$.

For the proof of the main result we need some preliminary results with which we now proceed. Let $X$ be a Banach space and $K$ be a bounded subset of $X$. The set $K$ is said to be weakly precompact (or conditionally weakly compact) if any sequence in $K$ has a weakly Cauchy subsequence. According to Rosenthal's celebrated $l^{1}$-Theorem [57], the unit ball $X_{1}$ of $X$ is weakly precompact if and only if $X$ does not contain a copy of $l^{1}$. And, a bounded linear operator $T$ from $X$ into a Banach space $Y$ is said to be weakly precompact if and only if the set $T\left(X_{1}\right)$ is weakly precompact.

Lemma 6.1. Let $A$ be an Arens regular Banach algebra with a BAI (= bounded approximate identity) $\left(e_{\alpha}\right)_{\alpha \in I}$. Then 
(a) $A A^{*}=A^{*} A=A^{*}$, where $A A^{*}=\left\{a f: a \in A, f \in A^{*}\right\}$ and $A^{*} A=\{f a$ : $\left.a \in A, f \in A^{*}\right\}$.

(b) For each $f$ in $A^{*},\left\|e_{\alpha} f-f\right\| \rightarrow 0$.

Proof. (a) For the proof of this assertion, see for instance [69, Theorem 3.1].

(b) To prove assertion (b), let $f$ be a functional in $A^{*}$. Then by (a), $f=$ $a g$ for some $a$ in $A$ and $g$ in $A^{*}$. As $e_{\alpha} f=\left(a e_{\alpha}\right) g$ and $\left(e_{\alpha}\right)$ is a BAI, $\left\|e_{\alpha} f-f\right\| \leq\|g\|\left\|a e_{\alpha}-a\right\| \rightarrow 0$.

Lemma 6.2. Let $A$ be an Arens regular Banach algebra with a BAI. Then $A$ does not contain a copy of $l^{1}$ if and only if, for each $a$ in $A$, the multiplication operator $\tau_{a}: A \rightarrow A$ is weakly precompact.

Proof. (i) Assume $A$ does not contain an isomorphic copy of $l^{1}$. Then any sequence $\left(x_{n}\right)$ in $A_{1}$ has a weakly Cauchy subsequence $\left(x_{n_{k}}\right)$. Therefore, for each $a$ in $A$, the sequence $\left(a x_{n_{k}}\right)_{k}$ is also weakly Cauchy and the operator $\tau_{a}$ is weakly precompact.

(ii) Conversely, assume that, for each $a$ in $A$, the operator $\tau_{a}$ is weakly precompact. To prove that $A$ does not contain a copy of $l^{1}$ it is enough to prove that none of the separable subalgebras of $A$ contains a copy of $l^{1}$. Indeed, if $X$ is a separable subspace of $A$ isomorphic to $l^{1}$ then the subalgebra $B$ of $A$ generated by $X$ is also separable and contains a copy of $l^{1}$. As in Lemma 3.4 of [69] one can show that any separable subalgebra of $A$ is contained in a separable subalgebra of $A$ having a sequential BAI. Thus we can and do assume that the algebra $A$ itself is separable and has a sequential BAI $\left(e_{n}\right)_{n \in \mathbb{N}}$. By hypothesis, each operator $\tau_{e_{n}}$ is weakly precompact. Now let $\left(a_{n}\right)$ be a sequence in $A_{1}$. Then $\left(a_{n}\right)$ has a subsequence $\left(a_{n_{k}^{1}}\right)_{k}$ such that the sequence $\left(e_{1} a_{n_{k}^{1}}\right)_{k}$ is weakly Cauchy. Now, the operator $\tau_{e_{2}}$ being weakly precompact, the sequence $\left(a_{n_{k}^{1}}\right)_{k}$ has a subsequence $\left(a_{n_{k}^{2}}\right)_{k}$ such that the sequence $\left(e_{2} a_{n_{k}^{2}}\right)_{k}$ is weakly Cauchy... and so on. Let $\left(a_{n_{k}^{k}}\right)_{k}$ be the diagonal sequence of the sequences thus obtained. Then, for each $p=1,2, \ldots$, the sequence $\left(e_{p} a_{n_{k}^{k}}\right)_{k}$ is weakly Cauchy. Now let $f$ be a given functional in $A^{*}$ and $\varepsilon>0$ an arbitrary number. By the preceding lemma, $\left\|e_{n} f-f\right\| \rightarrow 0$. Choose an integer $p$ such that $\left\|e_{p} f-f\right\|<\varepsilon / 2$, and fix this $p$. Since the sequence $\left(e_{p} a_{n_{k}^{k}}\right)_{k}$ is weakly Cauchy, there exists a number $L$ in $\mathbb{C}$ (depending of course on $f$ ) and an integer $k_{0}$ such that, for $k \geq k_{0},\left\|f\left(e_{p} a_{n_{k}^{k}}\right)-L\right\|<\varepsilon / 2$. Then, for $k \geq k_{0}$,

$$
\begin{aligned}
\left\|f\left(a_{n_{k}^{k}}\right)-L\right\| & =\left\|f\left(a_{n_{k}^{k}}\right)-f\left(e_{p} a_{n_{k}^{k}}\right)+f\left(e_{p} a_{n_{k}^{k}}\right)-L\right\| \\
& \leq\left\|f-e_{p} f\right\|\left\|a_{n_{k}^{k}}\right\|+\left\|f\left(e_{p} a_{n_{k}^{k}}\right)-L\right\| \\
& \leq \frac{\varepsilon}{2}+\frac{\varepsilon}{2}=\varepsilon .
\end{aligned}
$$

This proves that, for each $f$ in $A^{*}$, the sequence $\left(f\left(a_{n_{k}^{k}}\right)\right)_{k}$ converges. That is, the sequence $\left(a_{n_{k}^{k}}\right)$ is weakly Cauchy. Hence, by Rosenthal's $l^{1}$-Theorem, $A_{1}$ is weakly precompact, and $A$ does not contain a copy of $l^{1}$.

Remark 6.3. Let $G$ be an infinite compact group and $A=L^{1}(G)$ be the group algebra of $G$. Then $A$ has a BAI and for each $a$ in $A$, the operator $\tau_{a}$ is compact, see [69, Proposition 3.14] for instance. However, every closed nonreflexive subspace of $L^{1}(G)$, being weakly sequentially complete, contains, 
again by Rosenthal's $l^{1}$-Theorem, an isomorphic copy of $l^{1}$. This remark shows that without Arens regularity the preceding lemma may fail.

The next theorem draws a fine line between dual $C^{*}$-algebra and scattered $C^{*}$-algebras.

Theorem 6.4. Let $A$ be a $C^{*}$-algebra. Then we have:

(a) $A$ is a dual algebra if and only if, for each $a$ in $A$, the operator $\tau_{a}$ is weakly compact.

(b) $A^{*}$ has the RNP if and only if, for each $a$ in $A$, the operator $\tau_{a}$ is weakly precompact.

(c) $A^{*}$ has the Schur property if and only if, for each $a$ in $A$, the operator $\tau_{a}$ is weakly precompact and $A$ has the DPP.

Proof. The equivalence in (a) is well known [62, p. 157]. The equivalence in (b) follows from the preceding lemma and Corollary VII.10 of [28]. The equivalence in (c) follows from the preceding lemma and Theorem 3.6.

As explained in the introduction of this section, the two classes of $C^{*}$-algebras characterized by (a) and (b) of the preceding theorem are distinct even in the category of commutative $C^{*}$-algebras. To emphasize this we state the following corollary. However, since every commutative $C^{*}$-algebra has the DPP, in the category of commutative $C^{*}$-algebras, the class of $C^{*}$-algebras characterized by (b) and (c) are the same.

Corollary 6.5. Let $S$ be a locally compact topological space and $A=C_{0}(S)$. Then

(a) $S$ is discrete if and only if, for each $a$ in $A$, the operator $\tau_{a}$ is weakly compact.

(b) $S$ is dispersed if and only if, for each $a$ in $A$, the operator $\tau_{a}$ is weakly precompact.

The analogue of the preceding corollary for the group $C^{*}$-algebra $C^{*}(G)$ is Theorem 6.7 below. For the proof of this theorem we need the following lemma, see [50, Theorem 5] for the amenable case.

Lemma 6.6. Let $G$ be a locally compact group. Then the $C^{*}$-algebra $C^{*}(G)$ is a dual algebra if and only if $G$ is compact.

Proof. If $G$ is compact then $C^{*}(G)$ is a $C^{*}$-subalgebra of the dual algebra $K\left(L^{2}(G)\right)$ so that $C^{*}(G)$ is also a dual algebra. Conversely, assume $C^{*}(G)$ is a dual algebra. Then the algebra $C_{\rho}^{*}(G)$ is also a dual algebra since by [25, Proposition 1.15] $C_{\rho}^{*}(G)$ is isometrically isomorphic to a quotient of $C^{*}(G)$ and that a quotient of a dual algebra is also a dual algebra. But then, by Theorem 3 of [30], $G$ is compact.

Theorem 6.7. Let $G$ be a locally compact group and $A=C^{*}(G)$. Then

(a) $G$ is compact if and only if, for each $a$ in $A$, the operator $\tau_{a}$ is weakly compact.

(b) $G$ is an [AU]-group if and only if, for each $a$ in $A$, the operator $\tau_{a}$ is weakly precompact.

Proof. The equivalence in (a) is nothing but the preceding lemma. The equivalence in (b) follows from Theorem 6.4(b) above and Theorem 4.2 of [66].

Here we remark that if $G$ is the Fell group and $A=C^{*}(G)$ then, for each $a$ in $A$, the operator $\tau_{a}$ is weakly precompact but not every $\tau_{a}$ is weakly compact, 
see [66, p. 189]. We also remark that by Theorem 4.2 and by assertion (c) of Theorem 6.4 above, [COMPACT $]=[\mathrm{MOORE}] \cap[\mathrm{AU}]$.

\section{Arens Regularity of the algebra $C^{*}(G) \hat{\otimes} A$ AND THE RNP ON $B(G)$}

In [71] it was shown that the projective tensor product of two commutative $C^{*}$-algebras is Arens regular. The question whether the projective tensor product of two noncommutative $C^{*}$-algebras is Arens regular or not was left open. In this section, we give some results which prove in the negative several questions of the paper [71]. In particular, we show that the projective tensor product of even two von Neumann algebras need not be Arens regular. We apply our results to the projective tensor product of the algebra $C^{*}(G)$ with an arbitrary $C^{*}$-algebra $A$ and to several problems about geometric properties of the projective tensor product of two $C^{*}$-algebras.

7(a) Arens regularity of projective tensor products. In this subsection we shall study Arens regularity of the projective tensor product of two $C^{*}$-algebras. Our basic tools are the following definition and theorem which we have borrowed from the paper [71].

Definition 7.1. Let $A, B$ be two Banach algebras and $m: A \times B \rightarrow \mathbb{C}$ be a bounded bilinear form. The bilinear form $m$ is said to be biregular if, for any two pairs of sequences $\left(a_{i}\right),\left(\tilde{a}_{j}\right)$ in $A_{1}$ and $\left(b_{i}\right),\left(\tilde{b}_{j}\right)$ in $B_{1}$,

$$
\lim _{i} \lim _{j} m\left(a_{i} \tilde{a}_{j}, b_{i} \tilde{b}_{j}\right)=\lim _{j} \lim _{i} m\left(a_{i} \tilde{a}_{j}, b_{i} \tilde{b}_{j}\right)
$$

whenever these limits exist.

Theorem 7.2. Let $A$ and $B$ be two Banach algebras. Then the algebra $A \hat{\otimes} B$ is Arens regular if and only if every bounded bilinear form $m: A \times B \rightarrow \mathbb{C}$ is biregular.

To study Arens regularity of the projective tensor product of two $C^{*}$-algebras we need some preliminary results with which we proceed. The proof of the next lemma is very similar to that of Lemma 3.5 above and can be safely omitted. Throughout this section the abbreviation rwc will stand for relatively weakly compact.

Lemma 7.3. Let $A$ be a $C^{*}$-algebra and $f$ a functional in $A^{*}$. Then the set $H(f)=\left\{a^{* *} f b^{* *}: a^{* *}, b^{* *} \in A_{1}^{* *}\right\}$ is rwc if and only if the set $D(f)=\{a f b$ : $\left.a, b \in A_{1}\right\}$ is $r w c$.

The relative weak compactness of the set $H(f)$ for each $f$ in $A^{*}$ is very closely connected, in fact, as we shall see below, equivalent to the finiteness of the von Neumann algebra $A^{* *}$. To this end we shall need the following lemma, which is of independent interest.

Lemma 7.4. Let $M$ be a von Neumann algebra and $M_{*}$ be its predual. For a functional $f$ in $M_{*}$, if the set $U(f)=\left\{u_{1} f u_{2}: u_{1}, u_{2}\right.$ selfadjoint and unitary $\}$ is rwc then the set $H(f)=\left\{a f b: a, b \in M_{1}\right\}$ is rwc.

Proof. Assume the set $U(f)$ is rwc. To prove that the set $H(f)$ is rwc it is enough to prove that the set $H^{s}(f)=\left\{a f b: a, b \in M_{1}, a, b\right.$ are selfadjoint $\}$ is 
rwc. Indeed, if $a, b$ are in $M_{1}, a=a_{1}+i a_{2}, b=b_{1}+i b_{2}, a_{k}, b_{k} \quad(k=1,2)$ are selfadjoint, then $a f b=a_{1} f b_{1}+i a_{1} f b_{2}+i a_{2} f b_{1}-a_{2} f b_{2}$ so that $H(f)$ is the sum of four sets of the form $H^{s}(f)$. Now let $M_{1}^{s}=\left\{x \in M_{1}: x=x^{*}\right\}$. Then the set of the extreme points of $M_{1}^{s}$ is $U^{s}$, the set of selfadjoint unitary elements of $M_{1}^{s}$, see [60, p. 12]. Each $a$ in $M_{1}^{s}$ is the limit, by the KreinMilman Theorem, of a net $\left(a_{\alpha}\right)$ of $\mathrm{Co} U^{s}$ in the ultraweak topology. Hence, if $u_{2}$ is in $U^{s}, a_{\alpha} f u_{2} \rightarrow a f u_{2}$ weakly. Consequently, the set $\left\{a f u_{2}: a \in M_{1}^{s}\right\}$ is contained in $\overline{\mathrm{Co}} U(f)$, the weak closure of $\mathrm{Co} U^{s}$. A similar argument shows that $H(f) \subseteq \overline{\mathrm{Co}} U(f)$ so that the set $H(f)$ is rwc.

Corollary 7.5. Let $M$ be a von Neumann algebra and $M_{*}$ be its predual. Then $M$ is finite if and only if, for each $f$ in $M_{*}$, the set $H(f)=\left\{a f b: a, b \in M_{1}\right\}$ is rwc.

Proof. Assume $M$ is finite. Then, for each $f$ in $M_{*}$, the set $K=\left\{u f u^{*}: u \in\right.$ $M, u$ unitary $\}$ is rwc (see [74]). Let $f \in M_{*}$ be given. To prove that the set $H(f)$ is rwc we can assume that $f$ is positive. Then the set $K$ is contained in $M_{*}^{+}$and by Proposition 5.12 of $\left[62\right.$, p. 156] the set $\left\{a \varphi: a \in M_{1}\right.$ and $\left.\varphi \in K\right\}$ is rwc. Now, for any two unitary elements $u_{1}$ and $u_{2}$ of $M$,

$$
u_{1} f u_{2}^{*}=u_{2} u_{2}^{*}\left(u_{2} f u_{2}^{*}\right)=a\left(u_{2} f u_{2}^{*}\right), \quad \text { with } a=u_{1} u_{2}^{*} \text {, }
$$

so that $u_{1} f u_{2}^{*}$ is in the set $\left\{a \varphi: a \in M_{1}, \varphi \in K\right\}$. It follows that the set $\left\{u_{1} f u_{2}^{*}: u_{1}, u_{2} \in M\right.$ and are unitary $\}$ is rwc. Hence, by the preceding lemma, the set $H(f)$ is rwc. The converse is trivial.

Theorem 7.6. Let $A$ be a $C^{*}$-algebra. Assume that the von Neumann algebra $A^{* *}$ is not finite. Then the algebras $A \hat{\otimes} A^{* *}$ and $A^{* *} \hat{\otimes} A^{* *}$ are not Arens regular. If, in addition, the algebra $A$ is unital, then the algebra $A \hat{\otimes} A$ is not Arens regular either.

Proof. Since the algebra $A^{* *}$ is not finite, for some $f$ in $A^{*}$, the set $D(f)=$ $\left\{a f b: a, b \in A_{1}\right\}$ is not rwc by the preceding corollary and Lemma 7.3. Therefore, by Grothendieck's Double Limit Criterion, there exist three sequences $\left(a_{i}\right),\left(b_{i}\right)$ and $\left(\tilde{a}_{j}\right)$ in $A_{1}$ such that the following iterated limits

$$
\lim _{i} \lim _{j}\left\langle a_{i} f b_{i}, \tilde{a}_{j}\right\rangle \text { and } \lim _{j} \lim _{i}\left\langle a_{i} f b_{i}, \tilde{a}_{j}\right\rangle
$$

exist and are distinct. Now introduce the bilinear form $m: A \times A^{* *} \rightarrow \mathbb{C}$ defined by $m\left(a, a^{* *}\right)=\left\langle f, a a^{* *}\right\rangle$. This bilinear form is bounded. Let 1 be the unit element of $A^{* *}$. Put $\tilde{b}_{j}=1$ for $i=1,2, \ldots$. Then

$$
m\left(a_{i} \tilde{a}_{j}, b_{i} \tilde{b}_{j}\right)=\left\langle f, a_{i} \tilde{a}_{j} b_{i}\right\rangle=\left\langle a_{i} f b_{i}, \tilde{a}_{j}\right\rangle
$$

It follows that the bilinear form $m$ is not biregular and, by Theorem 7.2, the algebra $A \hat{\otimes} A^{* *}$ is not Arens regular. Since the algebra $A \hat{\otimes} A^{* *}$ is naturally isometrically isomorphic to a subalgebra of $A^{* *} \hat{\otimes} A^{* *}[21$, Corollary 14, p. 238] the algebra $A^{* *} \hat{\otimes} A^{* *}$ is not Arens regular either. The proof shows that if $A$ is unital, then the algebra $A \hat{\otimes} A$ is not Arens regular either.

For an immediate application of this theorem, let $H$ be an infinite dimensional Hilbert space. Then as one can see immediately that the von Neumann algebra $B(H)$ of bounded linear operators on $H$ is not finite. Therefore the algebras $B(H) \hat{\otimes} B(H)$ and $K(H) \hat{\otimes} B(H)$ are not Arens regular. The algebra 
$K(H) \hat{\otimes} K(H)$ is not Arens regular either. However, since the algebra $K(H)$ is not unital, this does not follow from the preceding theorem; it follows from the next two results instead.

Proposition 7.7. Let $A$ be an arbitrary Banach algebra. Assume that the following condition (c) is satisfied: There exists a functional $f$ in $A^{*}$ and an element $e$ in $A$ such that the set $E(f)=\left\{a f(b e): a, b \in A_{1}\right\}$ is not rwc. Then the algebra $A \hat{\otimes} A$ is not Arens regular.

Proof. We proceed exactly as in the proof of the preceding theorem but instead of the unit element of $A^{* *}$ we use the element $e$.

We recall that, for a Banach space $X$, the algebra of compact linear operators $K(X)$ on $X$ is Arens regular if and only if the space $X$ is reflexive, see [75, Theorem 3] or [72, Theorem 1].

Lemma 7.8. For any infinite dimensional reflexive Banach space $X$ the algebra $K(X)$ satisfies the condition (c) of the preceding proposition.

Proof. Let $X$ be an infinite dimensional Banach space. Fix $x$ in $X$ and $x^{*}$ in $X^{*}$ arbitrarily with $\|x\|=\left\|x^{*}\right\|=1$. Let $f=x \otimes x^{*}$. Then $f$ is a functional on $K(X)$ and is defined by $\langle f, u\rangle=\left\langle u(x), x^{*}\right\rangle$. Choose an element $y^{*}$ in $X_{1}^{*}$ such that $\left\langle x, y^{*}\right\rangle=1$. Then the one dimensional operator $e=y^{*} \otimes x$ is in $K(X)$, and it is such that $e(x)=\left\langle x, y^{*}\right\rangle x=x$. Now, for $u, v$ and $w$ in $K(X)$,

$$
\begin{aligned}
\langle u f(v \circ e), w\rangle & =\left\langle x \otimes x^{*}, u \circ w \circ v \circ e\right\rangle=\left\langle x^{*}, u \circ w \circ v \circ e(x)\right\rangle \\
& =\left\langle u^{*}\left(x^{*}\right), w \circ v(x)\right\rangle=\left\langle u^{*}\left(x^{*}\right) \otimes v(x), w\right\rangle
\end{aligned}
$$

so that

$$
u f(v \circ e)=u^{*}\left(x^{*}\right) \otimes v(x) .
$$

Choosing for $u$ and $v$ appropriate one dimensional operators such as $e$, for any $z$ in $X_{1}$ and $z^{*}$ in $X_{1}^{*}$, we can find $u, v$ in $K(X)_{1}$ such that $u^{*}\left(x^{*}\right)=z^{*}$ and $v(x)=z$ so that

$$
\left\{u f(v \circ e): u, v \in K(X)_{1}\right\}=X_{1}^{*} \otimes X_{1}
$$

where $X_{1}^{*} \otimes X_{1}$ is set of the simple tensors of the form $z^{*} \otimes z$. Now a simple application of Double Limit Criterion will show that the set $X_{1}^{*} \otimes X_{1}$ is not a rwc subset of $K(X)^{*}$.

The next result is now clear from the preceding lemma and Proposition 7.7. At this point maybe we should remark that if $X$ is reflexive and has the approximation property, then $K(X)^{* *}=B(X)$, and that always $K(X)$ is a subalgebra of $B(X)$, see [21, Chapter VIII].

Corollary 7.9. Let $X$ be a Banach space. Then, one of the algebras $K(X) \hat{\otimes} K(X)$ or $K(X) \hat{\otimes} K(X)^{* *}$ of $K(X)^{* *} \hat{\otimes} K(X)^{* *}$ is Arens regular if and only if $X$ is finite dimensional.

After these general results we now return to the algebras $C^{*}(G)$ and $C_{\rho}^{*}(G)$. The next theorem follows directly from Theorem 4.2 and Theorem 7.6. 
Theorem 7.10. Let $G$ be a locally compact group. If $G$ is not a Moore group, then the algebras $C^{*}(G) \hat{\otimes} W^{*}(G)$ and $W^{*}(G) \hat{\otimes} W^{*}(G)$ are not Arens regular.

Theorem 7.11. Let $G$ be a locally compact group. Then the following are equivalent.

(a) $G$ is compact.

(b) The space $B(G)$ has the $R N P$ and, for any $C^{*}$-algebra $A$, the algebra $C^{*}(G) \hat{\otimes} A$ is Arens regular.

Proof. Assume first that $G$ is compact. Then the space $B(G)=C^{*}(G)^{*}$ has the Schur property and the RNP by Theorem 4.3. Let $A$ be any $C^{*}$-algebra. Then, any bounded linear operator $u: A \rightarrow B(G)$ is weakly compact by GrothendieckPisier-Haagerup inequality [38], so compact. Hence, by Theorem 4.5 of [71], the algebra $C^{*}(G) \hat{\otimes} A$ is Arens regular.

Conversely, if (b) is satisfied and $G$ is not compact, then the group $G$ is not a Moore group. Hence, by the preceding theorem, the algebra $C^{*}(G) \hat{\otimes} W^{*}(G)$ is not Arens regular, contradicting the hypothesis.

To continue further we need the following lemma:

Lemma 7.12. Let $G$ be a locally compact group and $f$ be a functional in $A(G)$. If the set $H(f)=\left\{a f b: a, b \in V N(G)_{1}\right\}$ is not $r c w$, then the set $\widetilde{H}(f)=$ $\left\{a^{* *} f b^{* *}: a^{* *}, b^{* *} \in C_{\rho}^{*}(G)_{1}^{* *}\right\}$ is not rwc either.

Proof. Since $A(G)$ is a closed subspace of $B_{\rho}(G)=C_{\rho}^{*}(G)^{*}$, the functional $f$ is in $C_{\rho}^{*}(G)^{*}$. Assume the set $\widetilde{H}(f)$ is rwc. Then the set $E(f)=\{a f b$ : $\left.a, b \in C_{\rho}^{*}(G)_{1}\right\}$, which is a subset of $A(G)$, is also rwc. Now let $a \in V N(G)_{1}$ and $b \in C_{\rho}^{*}(G)_{1}$. Then, by the Kaplansky Density Theorem [60, p. 22], there exists a net $\left(a_{\alpha}\right)$ in $C_{\rho}^{*}(G)_{1}$ that converges to $a$ in the ultraweak topology of $V N(G)$. Hence, for $x$ in $V N(G)$,

$$
\left\langle a_{\alpha} f b, x\right\rangle=\left\langle f, a_{\alpha} x b\right\rangle \rightarrow\langle f, a x b\rangle=\langle a f b, x\rangle .
$$

It follows that $a f b$ is in the weak closure $\overline{E(f)}$ of $E(f)$. Similarly if both $a$ and $b$ in $V N(G)_{1}$, then $a f b \in \overline{E(f)}$. Consequently $H(f)$ is contained in $\overline{E(f)}$, and so it is rwc as well. By contrapositive, this proves the lemma.

Theorem 7.13. Let $G$ be a locally compact group. Then the following two assertions are equivalent.

(a) $G$ is compact.

(b) The space $A(G)$ has the $R N P$ and the algebra $C_{\rho}^{*}(G) \hat{\otimes} V N(G)$ is Arens regular.

Proof. The implication (a) $\Rightarrow$ (b) holds by Theorem 7.11. To prove the converse implication, assume (b) holds but $G$ is not compact. Then the algebra $V N(G)$, which is atomic, is not finite (see Theorem 4.3). Hence, for some $f$ in $A(G)$, the set $\left\{a f b: a, b \in V N(G)_{1}\right\}$ is not rwc. It follows from the preceding lemma that the set $\left\{a^{* *} f b^{* *}: a^{* *}, b^{* *} \in C_{\rho}^{*}(G)_{1}^{* *}\right\}$ is not rwc. Hence, by Lemma 7.3, the set $H(f)=\left\{a f b: a, b \in C_{\rho}^{*}(G)_{1}\right\}$ is not rwc either. It follows that there exist three sequences $\left(a_{i}\right),\left(b_{i}\right)$ and $\left(\tilde{a}_{j}\right)$ in the unit ball of $C_{\rho}^{*}(G)$ for which the following iterated limits

$$
\lim _{i} \lim _{j}\left\langle a_{i} f b_{i}, \tilde{a}_{j}\right\rangle \text { and } \lim _{j} \lim _{i}\left\langle a_{i} f b_{i}, \tilde{a}_{j}\right\rangle
$$


exist and are distinct. Let 1 be the unit element of $V N(G)$, and put $\tilde{b}_{j}=1$, $j=1,2, \ldots$. Since $C_{\rho}^{*}(G) \subseteq V N(G)$ we can consider the sequence $\left(b_{i}\right)$ as a sequence in $V N(G)_{1}$. Now introduce the bilinear form

$$
m: C_{\rho}^{*}(G) \times V N(G) \rightarrow \mathbb{C}
$$

defined by $m(a, b)=\langle f, a b\rangle$. Then

$$
m\left(a_{i} \tilde{a}_{j}, b_{i} \tilde{b}_{j}\right)=\left\langle f, a_{i} \tilde{a}_{j} b_{i}\right\rangle=\left\langle a_{i} f b_{i}, \tilde{a}_{j}\right\rangle
$$

so that the bilinear form $m$ is not biregular, and the algebra $C_{\rho}^{*}(G) \hat{\otimes} V N(G)$ is not Arens regular.

The following corollary is now obvious.

Corollary 7.14. Let $G$ be a locally compact group. Then $G$ is compact if and only if the space $A(G)$ has the RNP and, for any $C^{*}$-algebra $A$, the algebra $C_{\rho}^{*}(G) \hat{\otimes} A$ is Arens regular.

7(b) Applications to related problems. In this subsection we shall consider some applications of the fact that the projective tensor product of two $C^{*}$ algebras need not be Arens regular and other results given in subsection (a) to some related problems.

(i) Let $A$ and $B$ be two $C^{*}$-algebras. According to Grothendieck-PisierHaagerup inequality [38], every bounded linear operator $u: A \rightarrow B^{*}$ (also $\left.u: B \rightarrow A^{*}\right)$ is weakly compact. This not being a characteristic property of $C^{*}$ algebras and in general the algebra $A \hat{\otimes} B$ not being a $C^{*}$-algebra, the following question arises naturally: Is every bounded linear operator $u: A \hat{\otimes} B \rightarrow(A \hat{\otimes} B)^{*}$ weakly compact? The answer to this question is negative. Indeed, to say that a Banach algebra $D$ is Arens regular is equivalent to saying that, for each functional $f$ in $D^{*}$, the linear operator $T_{f}: D \rightarrow D^{*}$ defined by $T_{f}(a)=a f$ is weakly compact [70]. Should every bounded linear operator $u: A \hat{\otimes} B \rightarrow$ $(A \hat{\otimes} B)^{*}$ be weakly compact, the algebra $A \hat{\otimes} B$ would be Arens regular, which is in general not the case. However we have the following positive result which is due to the fact that, for $G$ compact, the space $C^{*}(G)^{*}$ has the Schur property.

Proposition 7.15. Let $G$ be a compact group. Then the dual space of the space $C^{*}(G) \hat{\otimes} C^{*}(G)$ has the Schur property and every bounded linear operator $u$ : $C^{*}(G) \hat{\otimes} C^{*}(G) \rightarrow\left(C^{*}(G) \hat{\otimes} C^{*}(G)\right)^{*}$ is compact.

Proof. The fact that the space $\left(C^{*}(G) \hat{\otimes} C^{*}(G)\right)^{*}$ has the Schur property follows from Corollary 3.4 of [58]. Since this space has the Schur property, the space $C^{*}(G) \hat{\otimes} C^{*}(G)$ does not contain a copy of $l^{1}[19$, Theorem 3, p. 22]. Therefore, by Rosenthal's $l^{1}$-Theorem [57], every bounded linear operator $u$ from the space $C^{*}(G) \hat{\otimes} C^{*}(G)$ into its dual is weakly compact and so compact.

(ii) Another question that arises about the algebra $A \hat{\otimes} B$ is this: To be sure, the algebra $A \hat{\otimes} B$ is not a $C^{*}$-algebra. However, keeping its projective tensor norm but defining a new multiplication on it, can we make it into a $C^{*}$-algebra? Again Arens regularity consideration shows that this is not possible. Indeed, should the space $A \hat{\otimes} B$ be a $C^{*}$-algebra for some multiplication, every bounded linear operator from $A \hat{\otimes} B$ into its dual would be weakly compact. But then, for its natural multiplication, the algebra $A \hat{\otimes} B$ would be Arens regular, which is not the case. 
(iii) Paper [71] contains several examples of biregular bilinear forms which are not Arens regular. However, if $A$ and $B$ are two unital Banach algebras then any bounded biregular bilinear form $m: A \times B \rightarrow \mathbb{C}$ is Arens regular. The converse of this question was left open [71, Question 3]. Now let $A$ be a unital $C^{*}$-algebra such that the algebra $A \hat{\otimes} A$ is not Arens regular. Then there exists at least one bounded bilinear form $m: A \times A \rightarrow \mathbb{C}$ which is not biregular. However, by Grothendieck-Pisier-Haagerup inequality [38], the bilinear form $m$, being weakly compact, is Arens regular. Thus, in general, a regular bilinear form need not be biregular nor conversely.

(iv) The results presented below are not direct applications of the fact that the projective tensor product of two $C^{*}$-algebras need not be Arens regular. However, as there is a unity in the method used in the proofs of these results and in those given in subsection (a), and also because these results are obtained as applications of the results given in subsection (a), we have found it appropriate to include them here.

The results presented below are about strictly (or doubly) weakly almost periodic functions, see [40 and 9]. Let $G$ be a locally compact group and $f$ a function in $C(G)$, the space of continuous, bounded complex-valued functions on $G$. For $x, g, h$ in $G$, write $f_{g}^{h}(x)=f(g x h)$. Then the function $f$ is said to be strictly weakly almost periodic if the set $\left\{f_{g}^{h}: h, g \in G\right\}$ is a rwc subset of $C(G)$. The space of the strictly weakly almost periodic functions on $G$ is denoted by $W S(G)$. The space $W S(G)$ is a translation invariant $C^{*}$-subalgebra of the space $W(G)$ of weakly almost periodic functions and it contains the space of the almost periodic functions on $G$ [9]. However, as shown to us by Ching Chou (private communication), who called such functions doubly weakly almost periodic, if $G$ is the motion group, then $W S(G) \cap C_{0}(G)=\{0\}$. Recently Hansel and Troallic [40, Theorem 4.3] showed that $C_{0}(G) \subseteq W S(G)$ if and only if $G$ is a [SIN]-group. The following proposition is an improvement and a short proof of one direction of this result.

Proposition 7.16. If $G$ is a [SIN]-group then, for each $f$ is $A(G)$, the set $\left\{f_{g}^{h}: g, h \in G\right\}$ is a rwc subset of $A(G)$. In particular, $C_{0}(G) \subseteq W S(G)$.

Proof. Assume $G$ is a [SIN]-group. Then the von Neumann algebra $V N(G)$ is finite $[22,13.10 .5]$. The first statement of the proposition is now a consequence of Corollary 7.5. The second statement also follows from the fact that $A(G)$ is dense in $C_{0}(G)$ and, for $f$ in $A(G),\|f\|_{A(G)} \geq\|f\|_{\infty}$.

The following result is an analogue of Theorem 4.3 of [40] for [IN]-groups.

Proposition 7.18. Let $G$ be a locally compact group. Then the following assertions are equivalent.

(a) $G$ is an $[I N]$-group.

(b) There exists $f$ in $A(G), f \neq 0$, such that the set $\left\{f_{g}^{h}: h, g \in G\right\}$ is rwc in $A(G)$.

(c) There exists $f$ in $C_{0}(G), f \neq 0$, such that the set $\left\{f_{g}^{g^{-1}}: g \in G\right\}$ is $r w c$ in $C_{0}(G)$.

Proof. (a) $\Rightarrow$ (b). First observe that if $f$ is in $A(G)$, then the set $O(f)=$ $\left\{a f b: a, b \in V N(G)_{1}\right\}$ is rwc in $A(G)$ if and only if, for any sequences 
$\left(a_{i}\right),\left(b_{i}\right)$ and $\left(c_{i}\right)$ in $V N(G)_{1}$,

$$
\lim _{i} \lim _{j}\left\langle a_{i} f b_{i}, c_{j}\right\rangle=\lim _{j} \lim _{i}\left\langle a_{i} f b_{i}, c_{j}\right\rangle
$$

whenever these limits exist. Now assume that $G$ is an [IN]-group. Then there exists $f$ in $A(G)$ which is a tracial state, i.e., $f$ is positive definite, $\|f\|=1$ and $\langle f, x y\rangle=\langle f, y x\rangle$ for all $x, y$ in $V N(G)$. For such an $f$,

$$
\left\langle a_{i} f b_{i}, c_{j}\right\rangle=\left\langle f, a_{i} c_{j} b_{i}\right\rangle=\left\langle f, b_{i} a_{i} c_{j}\right\rangle=\left\langle\left(b_{i} a_{i}\right) f, c_{j}\right\rangle .
$$

On the other hand, the algebra $V N(G)$ being Arens regular, the set $\{a f: a \in$ $\left.V N(G)_{1}\right\}$ is rwc in $A(G)$. Now the Double Limit Criterion shows that, for any tracial state $f$ in $A(G)$, the set $D(f)$ is rwc in $A(G)$.

The implication (b) $\Rightarrow(\mathrm{c})$ is trivial. To prove the implication $(\mathrm{c}) \Rightarrow(\mathrm{a})$, assume for some $f$ in $C_{0}(G), f \neq 0$, the set $\left\{f_{g}^{g^{-1}}: g \in G\right\}$ is rwc in $C_{0}(G)$. For some $a$ in $G, f(a) \neq 0$. Replacing $f$ by $f_{a^{-1}}$ and rescaling if necessary, we can assume that $f(e)=1$. Let $K$ be the closed convex hull of the set $\left\{f_{g}^{g^{-1}}: g \in G\right\}$. Then $K$ is a weakly compact subset of $C_{0}(G)$ and $k(e)=1$ for each $k$ in $C_{0}(G)$. Consider the distal affine weakly continuous action of the (discrete) group $G$ on $K$ defined by

$$
G \times K \rightarrow K, \quad(g, k) \mapsto k_{g}^{g^{-1}} .
$$

Then, by the Ryll-Nardzewski Fixed Point Theorem [36], there exists $k$ in $K$ such that $k_{g}^{g^{-1}}=k$ for all $g$ in $G$. Let now

$$
V=\{g \in G:|k(g)-k(e)| \leq 1 / 2\} .
$$

Then $V$ is a compact neighbourhood of $e$ with $g^{-1} V g=V$ for all $g$ in $G$. Hence $G$ is an [IN]-group, and the implication (c) $\Rightarrow($ a) holds.

\section{The Algebras $A_{p}(G)$ AND $P M_{p}(G)$}

In this section we shall present some results about Arens regularity of the algebras $A_{p}(G), P F_{p}(G), P M_{p}(G), 1<p<\infty$; some results about almost periodic functionals on these algebras; and, some results about invariant subalgebras of the algebras $A_{p}(G)$ and $P M_{p}(G)$. Note that for $p \neq 2$, the theory of operator algebras (which we have benefited in earlier chapters) will no longer be applicable.

8(a) Preliminaries. In addition to the preliminary results and notations given in $\S 2$ we shall need some results and notations about the algebras $A_{p}(G)$ and $P M_{p}(G)$ with which we start. Let $G$ be a locally compact group equipped with a fixed left Haar measure. The spaces $L^{p}(G)(1 \leq p \leq \infty)$ have their usual meaning. We recall that, for any function $f: G \rightarrow \mathbb{C}, f^{\vee}$ and $\tilde{f}$ are defined by $f^{\vee}(x)=f\left(x^{-1}\right)$ and $\tilde{f}(x)=\overline{f\left(x^{+1}\right)}$. For any two measurable functions $f$ and $g$ on $G$, their convolution $f * g$ is defined by

$$
f * g(x)=\int_{G} g\left(y^{-1} x\right) f(y) d y
$$

whenever this makes sense. For each $x$ in $G$, the operator $\lambda(x): L^{p}(G) \rightarrow$ $L^{p}(G)$ defined by $\lambda(x)(f)=x^{-1} f$, where $x^{-1} f(y)=f\left(x^{-1} y\right)$, is the left 
translation operator on $L^{p}(G)$. For $f$ in $L^{1}(G)$, the operator $\rho(f): L^{p}(G) \rightarrow$ $L^{p}(G)$ is defined by $\rho(f)(g)=f * g . \rho(f)$ is a bounded linear operator on $L^{p}(G)$ and by $\|\rho(f)\|$ we shall always denote the operator norm of $\rho(f)$. For $1<p<\infty$, the Figà-Talamanca-Herz algebra $A_{p}(G)$ is the space of functions $f: G \rightarrow \mathbb{C}$ which can be represented, nonuniquely, as

$$
f=\sum_{n=1}^{\infty} v_{n} * u_{n}^{\vee}
$$

with $u_{n}$ in $L^{p}(G), v_{n}$ in $L^{q}(G)\left(\frac{1}{p}+\frac{1}{q}=1\right)$ and $\sum_{n=1}^{\infty}\left\|u_{n}\right\|_{p}\left\|v_{n}\right\|_{q}<\infty$. The norm of $f$ is defined by

$$
\|f\|_{A_{p}(G)}=\inf \sum_{n=1}^{\infty}\left\|u_{n}\right\|_{p}\left\|v_{n}\right\|_{q},
$$

where the infimum is taken over all the representations of $f$ as in $(*)$. It is known that $A_{p}(G)$ is a subspace of $C_{0}(G)$ and, equipped with the above norm and the pointwise multiplication is a regular tauberian algebra whose Gelfand spectrum is $G$. Furthermore the algebra $A_{p}(G)$ has a BAI (= bounded approximate identity) if and only if the group $G$ is amenable. For $p=2$, $A_{p}(G)=A(G)$, the Fourier algebra of $G$. (See [56].)

Each element $f$ of $L^{1}(G)$ defines a bounded functional $\phi_{f}$ on $A_{p}(G)$ by

$$
\left\langle\phi_{f}, u\right\rangle=\int_{G} f(x) u(x) d x .
$$

The norm of $\phi_{f}$ as an element of $A_{p}(G)^{*}$ and the operator norm of $\rho(f)$ are the same. That is,

$$
\left\|\phi_{f}\right\|=\sup _{\|u\|_{A_{p}(G)} \leq 1}\left|\left\langle\phi_{f}, u\right\rangle\right|=\|\rho(f)\|=\sup _{\|g\|_{p} \leq 1}\|f * g\|_{p} .
$$

It follows that $L^{1}(G)$ can be considered as a subspace of $A_{p}(G)^{*}$. By definition, $P F_{p}(G)$ and $P M_{p}(G)$ are the closures of $L^{1}(G)$ in $A_{p}(G)^{*}$ with respect to the norm and weak* topologies of $A_{p}(G)^{*}$. Since $A_{p}(G)$ is a Banach algebra, the spaces $P M_{p}(G)$ and $P F_{p}(G)$ are $A_{p}(G)$-modules. The module actions are defined, for $u, v$ in $A_{p}(G)$ and $T$ in $P M_{p}(G)$ or $P F_{p}(G)$, by $\langle u T, v\rangle=$ $\langle T, u v\rangle$. Let $W_{p}(G)=P F_{p}(G)^{*}$. Then the space $W_{p}(G)$ has been identified as a subalgebra of $C(G)$, the space of continuous bounded functions on $G$. The space $W_{p}(G)$ also, equipped with the pointwise multiplication and the dual norm, is a commutative Banach algebra. Also, the following inclusions

$$
A_{p}(G) \subseteq W_{p}(G) \subseteq B_{p}(G)
$$

hold, where $B_{p}(G)$ is the pointwise multiplier of the algebra $A_{p}(G)$. Furthermore $B_{p}(G)$ equipped with the multiplier norm

$$
\|u\|=\sup \left\{\|u v\|_{A_{p}(G)}:\|v\|_{A_{p}(G)} \leq 1\right\}
$$

is isometrically isomorphic to the algebra $W_{p}(G)$ if and only if the group $G$ is amenable, see [15] for details. For $p=2, P F_{2}(G)=C_{\rho}^{*}(G)$ and $P M_{2}(G)=$ $V N(G)$. Finally, $U C_{p}(\widehat{G})$ denotes the norm closure in $P M_{p}(G)$ of the subspace $A_{p}(G) P M_{p}(G)=\left\{u T: u \in A_{p}(G), T \in P M_{p}(G)\right\}$ of $P M_{p}(G)$. If $G$ is amenable, then the algebra $A_{p}(G)$ has a bounded approximate identity and, by 
the Cohen-Hewitt Factorization Theorem [42, 32.22], the space $A_{p}(G) P M_{p}(G)$ is closed. The space $W_{p}(\widehat{G})$ denotes the subspace of $P M_{p}(G)$ consisting of the weakly almost periodic functionals on $A_{p}(G)$, i.e., $W_{p}(\widehat{G})=\operatorname{wap}\left(A_{p}(G)\right)$. We refer readers to [15 and 56] for details.

8(b) Arens regularity of the algebras $A_{p}(G), P F_{p}(G)$ and almost periodic functionals on $P M_{p}(G)$. Recently B. Forrest has proved the following results, see [26].

(a) If the algebra $A_{p}(G)$ is Arens regular, then the group $G$ is discrete.

(b) The group $G$ is discrete if and only if the algebra $A_{p}(G)$ is a (two-sided) ideal in its second dual $A_{p}(G)^{* *}$ equipped with either Arens product.

On the other hand, the algebra $A_{p}(G)$ being a regular tauberian algebra its Gelfand spectrum is $G$ and $G$ separates the points of $A_{p}(G)$. In view of these facts the following theorem, which is essentially contained in Theorem 2.3 of [23], is relevant here. In this theorem, by $\Phi$ we denote the Gelfand spectrum of $A^{*}$ and by $\overline{A A^{*}}$ and $\overline{\text { span } \Phi}$ we denote the norm closures of the spaces $A A^{*}$ and $\operatorname{span} \Phi$.

Theorem 8.1. Let $A$ be a regular tauberian algebra which is an ideal in its second dual. Then

(a) The space $\overline{A A^{*}}$ is contained in $\overline{\text { span }} \Phi$.

(b) If $A$ also has a BAI (bounded approximate identity), then $A$ is Arens regular if and only if $A^{*}=\overline{\operatorname{span}} \Phi$. Moreover, in this case, the space $A^{*}$ has the RNP.

Proof. The proof uses in an essential way the fact that the set $\Phi$ separates the points of $A$.

(a) Let $a$ in $A$ and $f$ in $A^{*}$ be two arbitrary elements. We have to show that the functional af is in $\overline{\operatorname{span}} \Phi$. Assume this is not the case. Then, by the Hahn-Banach Theorem, there exists an element $a^{* *}$ in $A^{* *}$ such that $\left\langle a f, a^{* *}\right\rangle=\left\langle f, a a^{* *}\right\rangle \neq 0$ but $\left\langle g, a^{* *}\right\rangle=0$ for all $g$ in $\overline{\operatorname{span}} \Phi$. Let $g$ be an arbitrary element of $\Phi$. Then $a g=g(a) g$ is in $\operatorname{span} \Phi$ so that $\left\langle a g, a^{* *}\right\rangle=$ $g(a)\left\langle g, a^{* *}\right\rangle=0$. That is, $\left\langle g, a a^{* *}\right\rangle=0$. This being true for all $g$ in $\Phi$ and $\Phi$ separating the points of $A, a a^{* *}=0$. But then $\left\langle f, a a^{* *}\right\rangle=0$ also, contradicting the fact that $\left\langle f, a a^{* *}\right\rangle \neq 0$. This proves that $\overline{A A^{*}} \subseteq \overline{\operatorname{span}} \Phi$.

(b) Assume $A$ has a BAI. Then the space $A A^{*}$ is closed in $A^{*}$. Now, if $A$ is Arens regular, then $A^{*}=A A^{*} \subseteq \overline{\operatorname{span}} \Phi$, see [69, Corollary 3.2]. Hence $A^{*}=\overline{\text { span }} \Phi$. Conversely, if $A^{*}=\overline{\text { span }} \Phi$, then obviously $A$ is Arens regular and $A^{*}=a p(A)=\overline{\operatorname{span}} \Phi$. The last assertion follows from Corollary 3.7 of [69].

Corollary 8.2 [33, p. 15]. If the group $G$ is discrete, then $U C_{p}(\widehat{G})=\overline{\operatorname{span}} \Phi$, where $\Phi=\left\{\delta_{x}: x \in G\right\}$ and $\delta_{x}: A_{p}(G) \rightarrow \mathbb{C}$ is defined by $\delta_{x}(u)=u(x)$.

Proof. By the preceding theorem and Forrest's result recalled above, $U C_{p}(\widehat{G}) \subseteq$ $\overline{\operatorname{span}} \Phi$. Also if $x \in G, \phi \in A_{p}(G)$ such that $\phi(x)=1$, then $\phi \cdot \delta_{x}=\delta_{x}$. Hence $\delta_{x} \in U C_{p}(\widehat{G})$. Consequently $U C_{p}(\widehat{G})=\overline{\operatorname{span}} \Phi$.

The next result summarizes what we know about Arens regularity of the algebra $A_{p}(G)$ when $G$ is amenable. This results follows from the above theorem and corollary. 
Corollary 8.3. Let $G$ be an amenable locally compact group. Then the following assertions are equivalent.

(a) The algebra $A_{p}(G)$ is Arens regular.

(b) The equality $P M_{p}(G)=P F_{p}(G)$ holds.

(c) The equality $P M_{p}(G)=\overline{\text { span }} \Phi$ holds.

Furthermore any one of these conditions implies that $G$ is discrete.

However we were unable to prove or disprove that the equality $P M_{p}(G)=$ $\overline{\operatorname{span}} \Phi$ implies that $G$ is finite, except for $p=2$ [51, Proposition 5.3].

Now we return to the algebras $P F_{p}(G)$ and $P M_{p}(G)$. We shall see that, when $G$ is compact, the algebra $P F_{p}(G)$ behaves very much like the $C^{*}$-algebra $C^{*}(G)$ in the sense that, for each $T$ in $P F_{p}(G)$, the multiplication operator $S \mapsto T \circ S$ (also $S \mapsto S \circ T$ ) on $P F_{p}(G)$ is compact and that $P F_{p}(G)^{*}=$ $W_{p}(G)=a p\left(P F_{p}(G)\right)$ so that the algebra $P F_{p}(G)$ is Arens regular and the space $W_{p}(G)$ has the RNP. The algebra $P M_{p}(G)$ being a more complicated space, we have only partial results about (weakly) almost periodic functionals on it. The problem of Arens regularity of the algebra $P M_{p}(G)$ is closely connected with that of the operator algebra $B\left(L^{p}(G)\right)$, which is a long standing open question. Before proving these results we first recall some facts about the algebras $P M_{p}(G)$ and $A_{p}(G)$ we shall need below.

The two spaces $P F_{p}(G)$ and $P M_{p}(G)$ are considered as subalgebras of the operator algebra $B\left(L^{p}(G)\right)$ of continuous bounded linear operators on $L^{p}(G)$. As is well known, $B\left(L^{p}(G)\right)=\left(L^{p}(G) \hat{\otimes} L^{q}(G)\right)^{*}\left(\frac{1}{p}+\frac{1}{q}=1\right)$. Let

$$
N=\left\{f=\sum_{n=1}^{\infty} u_{n} \otimes v_{n} \in L^{p}(G) \hat{\otimes} L^{q}(G): \sum_{n=1}^{\infty} v_{n} * v_{n}^{\vee}=0 \text { on } G\right\} .
$$

Then $N$ is a closed subspace of $L^{p}(G) \hat{\otimes} L^{q}(G)$ and $A_{p}(G)$, as a Banach space, is isometrically isomorphic to the quotient space $L^{p}(G) \hat{\otimes} L^{q}(G) / N$. The mapping

$$
P: L^{p}(G) \hat{\otimes} L^{q}(G) \rightarrow A_{p}(G)
$$

defined by $P(u \otimes v)=v * u^{\vee}$ is a bounded onto linear operator whose kernel is $N$. Therefore, as a Banach space, $P M_{p}(G)$ can be identified to the annihilator $N^{\perp}$ of $N$ in $B\left(L^{p}(G)\right)$. It follows that, for a net $\left(T_{\alpha}\right)$ in $P M_{p}(G)$, to say that $T_{\alpha} \rightarrow T$ in the weak*-topology $\sigma\left(P M_{p}(G), A_{p}(G)\right)$ of $P M_{p}(G)$ is equivalent to saying that $T_{\alpha} \rightarrow T$ in the weak*-topology (or weak operator topology) $\sigma\left(B\left(L^{p}(G)\right), L^{p}(G) \hat{\otimes} L^{q}(G)\right)$ of $B\left(L^{p}(G)\right)$. We shall use this fact without further comment.

Lemma 8.4. For any locally compact group $G$ and $1<p<\infty$, the inclusion $L^{p}(G) \hat{\otimes} L^{q}(G) \subseteq w a p\left(B\left(L^{p}(G)\right)\right)$ holds.

Proof. This is a particular case of a more general result. Indeed, if $A$ is any Arens regular Banach algebra, then, as one can see very easily, $A^{*} \subset w a p\left(A^{* *}\right)$. Now it is enough to remark that the operator algebra $A=K\left(L^{p}(G)\right)$ is Arens regular (see [72] or [75]) and $A^{* *}=B\left(L^{p}(G)\right)$, see [21, Chapter VIII].

Corollary 8.5. For any locally compact group $G$ and $1<p<\infty$, the inclusion $A_{p}(G) \subseteq w a p\left(P F_{p}(G)\right)$ holds.

Proof. This follows from the preceding lemma and the fact that $A_{p}(G)$ is a quotient of the space $L^{p}(G) \hat{\otimes} L^{q}(G)$. 
Whether the algebra $P M_{p}(G)$ is Arens regular or not is not known for $p \neq$ 2. A negative answer to this inquiry would show that the operator algebra $B\left(L^{p}(G)\right)$ is also irregular, which is not yet known.

Now let $X$ be a reflexive Banach space and $E$ be its closed unit ball equipped with the weak topology. Denote by $C(E, X)$ the Banach space of continuous functions $\varphi: E \rightarrow X$ equipped with the supremum norm. Then the space $K(X)$ of compact linear operators can naturally be identified with a closed subspace of $C(E, X)$. Therefore, by the vector-valued version of the Ascoli Theorem, a subset $D$ of $K(X)$ is relatively norm compact if and only if, for each $t$ in $E$, the set $D(t)=\{\varphi(t): \varphi \in D\}$ is relatively (norm) compact and the set $D$ is equicontinuous on $E$. The elements of $D$ being linear operators, $D$ is equicontinuous on $E$ if it is equicontinuous at zero and this is equivalent, as one can see easily using the definition of equicontinuity, to the following: For each weakly null sequence $\left(t_{n}\right)$ in $E, \lim _{n \rightarrow \infty} \sup _{\varphi \in D}\left\|\varphi\left(t_{n}\right)\right\|=0$. As an illustration of this remark we give the following result, which is of independent interest.

Lemma 8.6. Assume the group $G$ is compact and $1<p<\infty$. Then, on the algebra $P F_{p}(G)$, for each $T$ in $P F_{p}(G)$, the multiplication operator $S \mapsto S \circ T$ (also, $S \mapsto T \circ S$ ) is compact.

Proof. We first recall that the space $C(G)$ is dense in $L^{1}(G)$ and $P F_{p}(G)$ is the norm closure of $\left\{\rho(f): f \in L^{1}(G)\right\}$ in $B\left(L^{p}(G)\right)$. Here, as recalled above, $\rho(f): L^{p}(G) \rightarrow L^{p}(G)$ is the linear operator defined by $\rho(f)(g)=f * g$. This operator $\rho(f)$ being compact, the space $P F_{p}(G)$ is contained in $K\left(L^{p}(G)\right)$. Let $E$ be the unit ball of $L^{p}(G)$ equipped with the weak topology. It is clear that a subset $D$ of $P F_{p}(G)$ is relatively compact if and only if $D$ as a subset of $C\left(E, L^{p}(G)\right)$ is relatively compact. Now to prove that, for each $T$ in $P F_{p}(G)$, the multiplication operator $S \mapsto S \circ T$ is compact in $P F_{p}(G)$, it is enough to prove that, for each $f$ in $C(G)$, the set $\left\{S \circ \rho(f): S \in P F_{p}(G),\|S\| \leq 1\right\}$ is relatively compact in $P F_{p}(G)$. Fix an $f$ in $C(G)$. The set $\{S \circ \rho(f)$ : $\left.S \in P F_{p}(G),\|S\| \leq 1\right\}$ is contained in the norm closure of the set $\{\rho(g) \circ$ $\rho(f): g \in C(G),\|\rho(g)\| \leq 1\}$. Therefore it is enough to prove that the set $D=\{\rho(g * f): g \in C(G),\|\rho(g)\| \leq 1\}$ is relatively compact in $C\left(E, L^{p}(G)\right)$. Since for each $h$ in $L^{p}(G)$, the multiplication operator $\tau_{h}: L^{p}(G) \rightarrow L^{p}(G)$ defined by $\tau_{h}(k)=k * h$ is compact and the set $\{g * f: g \in C(G),\|\rho(g)\| \leq 1\}$ is bounded in $L^{p}(G)$, the set

$$
D(h)=\{g * f * h: g \in C(G),\|\rho(g)\| \leq 1\}
$$

is relatively compact in $L^{p}(G)$. Now let $\left(h_{n}\right)$ be a weakly null sequence in $E$. Then

$$
\sup _{\|\rho(g)\| \leq 1}\left\|g * f * h_{n}\right\|_{p} \leq \sup _{\|\rho(g)\| \leq 1}\left\|\rho(g)\left(f * h_{n}\right)\right\|_{p} \leq\left\|f * h_{n}\right\|_{p} \rightarrow 0
$$

since the multiplication operator $k \mapsto f * k$ from $L^{p}(G)$ to $L^{p}(G)$ is compact. Hence, by the remark above, we conclude that the set $D$ is relatively compact in $C\left(E, L^{p}(G)\right)$, and the multiplication operator $S \mapsto S \circ T$ is compact on $P F_{p}(G)$ for each $T$ in $P F_{p}(G)$.

The second statement of the next corollary is due to Granirer [31, Theorem 2]. 
Corollary 8.7. Assume the group $G$ is compact. Then $\operatorname{ap}\left(P F_{p}(G)\right)=W_{p}(G)$ and the space $W_{p}(G)$ has the RNP.

Proof. Let $\left(f_{\alpha}\right)_{\alpha \in I}$ be a BAI for the group algebra $L^{1}(G)$ with $\left\|f_{\alpha}\right\| \leq 1$ for all $\alpha$ in $I$. Then, for each $g$ in $L^{1}(G)$,

$$
\left\|\rho\left(f_{n}\right) \circ \rho(g)-\rho(g)\right\|=\sup _{\|h\|_{p} \leq 1}\left\|f_{n} * g * h-g * h\right\|_{p} \leq\left\|f_{n} * g-g\right\|_{1} \rightarrow 0 .
$$

Also $\left\|\rho\left(f_{n}\right)\right\| \leq\left\|f_{n}\right\|_{1} \leq 1$. Thus, since the space $\left\{\rho(f): f \in L^{1}(G)\right\}$ is norm dense in $P F_{p}(G)$, the net $\left(\rho\left(f_{\alpha}\right)\right)_{\alpha \in I}$ is a BAI for $P F_{p}(G)$. Now since the algebra $K\left(L^{p}(G)\right)$ is Arens regular, so is its closed subalgebra $P F_{p}(G)$. Hence, by [69, Corollary 3.2], $P F_{p}(G)^{*}=P F_{p}(G) P F_{p}(G)^{*}$. The preceding lemma shows that the space $P F_{p}(G) P F_{p}(G)^{*}$ is contained in $a p\left(P F_{p}(G)\right)$. It follows that $P F_{p}(G)^{*}=a p\left(P F_{p}(G)\right)$. The fact that the space $W_{p}(G)=P F_{p}(G)^{*}$ has the RNP follows from [69, Corollary 3.7].

For the next theorem we recall that if $T$ is in $P M_{p}(G)$ and $v * u^{\vee}$ is a simple element of $A_{p}(G)$ then $\left\langle T, v * u^{\vee}\right\rangle=\langle T(u), v\rangle$. And likewise, if $T$ is in $B\left(L^{p}(G)\right)$ and $u \otimes v$ is a simple element of $L^{p}(G) \hat{\otimes} L^{q}(G)$,

$$
\langle T, u \otimes v\rangle=\langle T(u), v\rangle .
$$

We also recall that, when the group $G$ is compact, the space $L^{p}(G)$ with the convolution as the multiplication is a Banach algebra and that this Banach algebra has an (unbounded) approximate identity. Moreover, as we have already used in the proof of Lemma 8.6, for each $f$ in $L^{p}(G)$ the multiplication operator $g \mapsto f * g$ (also $g \mapsto g * f$ ) is compact on $L^{p}(G)$.

Theorem 8.8. Let $G$ be a locally compact group. Then the following are equivalent.

(a) The group $G$ is compact.

(b) The inclusion $A_{p}(G) \subseteq$ ap $\left(P M_{p}(G)\right)$ holds.

(c) On the unit ball of $P M_{p}(G)$ multiplication is jointly continuous in the weak*-topology.

Proof. (a) $\Rightarrow(\mathrm{b})$. Assume $G$ is compact. Let $f=v * u^{\vee}$ be a simple element of $A_{p}(G)$. Let us first prove that the set

$$
E=\left\{f T: T \in P M_{p}(G),\|T\| \leq 1\right\}
$$

is a relatively compact subset of $A_{p}(G)$, i.e., $f$ is almost periodic on $P M_{p}(G)$. Here $f T$ is defined by $\langle f T, S\rangle=\langle f, S \circ T\rangle$. To this end, fix an element $h$ in $L^{p}(G)$. Then since $L^{p}(G) \subseteq L^{1}(G)$, the operator $\rho(h)$ is in $P M_{p}(G)$. Put $\phi=f \rho(h)$. Then $\phi$ is a functional in $A_{p}(G)$. Consider the set

$$
F=\left\{\phi T: T \in P M_{p}(G)_{1}\right\}=\left\{(f \rho(h)) T: T \in P M_{p}(G)_{1}\right\} .
$$

Let us see that the set $F$ is relatively compact in $A_{p}(G)$. To prove this it is enough to prove that, for any net $\left(S_{\alpha}\right)$ in the unit ball of $P M_{p}(G)$ that converges weak* to zero converges to zero uniformly on $F$, see, for instance, [46, (A), p. 148]. Let $\left(S_{\alpha}\right)$ be such a net. Then

$$
\begin{aligned}
\left\langle(f \rho(h)) T, S_{\alpha}\right\rangle & =\left\langle f \rho(h), S_{\alpha} \circ T\right\rangle=\left\langle f, S_{\alpha} \circ T \circ \rho(h)\right\rangle \\
& =\left\langle S_{\alpha} \circ T \circ \rho(h), v * u^{\vee}\right\rangle=\left\langle S_{\alpha} \circ T(h * u), v\right\rangle \\
& =\left\langle S_{\alpha}, T(h * u) \otimes v\right\rangle .
\end{aligned}
$$


But $T(h * u)=T(h) * u$ and the multiplication operator on $L^{p}(G)$ being compact, the set

$$
H=\left\{T(h) * u: T \in P M_{p}(G)_{1}\right\}
$$

is a relatively compact subset of $L^{p}(G)$. It follows that the set

$$
\widehat{H}=\left\{T(h * u) \otimes v: T \in P M_{p}(G)_{1}\right\}
$$

is a relatively compact subset of $L^{p}(G) \hat{\otimes} L^{q}(G)$. Since $S_{\alpha} \rightarrow 0$ in the weak* topology of $B\left(L^{p}(G)\right)$, we have

$$
\lim _{\alpha} \sup _{\|T\| \leq 1}\left\langle S_{\alpha}, T(h * u) \otimes v\right\rangle=0 .
$$

This proves that the set $F$ is relatively compact in $A_{p}(G)$ and hence the functional $\phi=f \rho(h)$ is almost periodic on $P M_{p}(G)$. Now, let $\left(h_{\alpha}\right)_{\alpha \in I}$ be an approximate identity in $L^{p}(G)$. Then, for $T$ in $P M_{p}(G)$,

$$
\begin{aligned}
\left\langle f \rho\left(h_{\alpha}\right), T\right\rangle & =\left\langle f, T \circ \rho\left(h_{\alpha}\right)\right\rangle=\left\langle T \circ \rho\left(h_{\alpha}\right), v * u^{\vee}\right\rangle \\
& =\left\langle T\left(h_{\alpha} * u\right), v\right\rangle \rightarrow\langle T(u), v\rangle=\left\langle T, v * u^{\vee}\right\rangle .
\end{aligned}
$$

That is, $f \rho\left(h_{\alpha}\right) \rightarrow f$ weakly in $A_{p}(G)$. The space $a p\left(P M_{p}(G)\right)$ being a closed subspace of $P M_{p}(G)^{*}$, we conclude that the functional $f=v * u^{*}$ is almost periodic on $P M_{p}(G)$. This proves that the set $E$ is relatively compact. This being true for simple elements of the form $v * u^{\vee}$ of $A_{p}(G)$ and that these simple elements being total in $A_{p}(G)$, we conclude that the inclusion $A_{p}(G) \subseteq$ $\operatorname{ap}\left(P M_{p}(G)\right)$ holds.

(b) $\Rightarrow$ (c). Assume that $A_{p}(G) \subseteq a p\left(P M_{p}(G)\right)$. Let $\left(T_{\alpha}\right)_{\alpha \in I}$ and $\left(S_{\alpha}\right)_{\alpha \in I}$ be two weak*-convergent nets in the unit ball of $P M_{p}(G)$ with $T=$ weak*- $\lim T_{\alpha}$ and $S=$ weak* $^{*} \lim S_{\alpha}$. Let $f=v * u^{\vee}$ be a simple element of $A_{p}(G)$. Then, by assumption, the set $\left\{f S_{\alpha}: \alpha \in I\right\}$ is a relatively compact subset of $A_{p}(G)$. Now, for any $W$ in $P M_{p}(G)$,

$$
\left\langle f S_{\alpha}, W\right\rangle=\left\langle f, W \circ S_{\alpha}\right\rangle \rightarrow\langle f, W \circ S\rangle=\langle f S, W\rangle .
$$

That is, $f S_{\alpha} \rightarrow f S$ weakly in $A_{p}(G)$. The set $\left\{f S_{\alpha}: \alpha \in I\right\}$ being relatively compact, we conclude that $f S_{\alpha} \rightarrow f S$ in the norm of $A_{p}(G)$. It follows that

$$
\lim _{\alpha}\left\langle T_{\alpha} \circ S_{\alpha}, f\right\rangle=\lim _{\alpha}\left\langle f S_{\alpha}, T_{\alpha}\right\rangle=\langle f S, T\rangle=\langle T \circ S, f\rangle .
$$

Again by the fact that the simple functions of the form $f=v * u^{\vee}$ is total in $A_{p}(G)$, we conclude that $T_{\alpha} \circ S_{\alpha} \rightarrow T \circ S$ in the weak* topology of $P M_{p}(G)$.

(c) $\Rightarrow\left(\right.$ a) . Assume (c) holds but $G$ is not compact. Let $\left(x_{\alpha}\right)$ be a net in $G$ that converges to infinity. The operators

$$
\lambda\left(x_{\alpha}\right): L^{p}(G) \rightarrow L^{p}(G)
$$

defined by $\lambda\left(x_{\alpha}\right) f=x_{\alpha}^{-1} f$, where $x_{\alpha}^{-1} f(x)=f\left(x_{\alpha}^{-1} x\right)$, are in the unit ball of $P M_{p}(G)$. Let $T$ be a weak*-cluster point of the net $\left(\lambda\left(x_{\alpha}\right)\right)$. Then, since $A_{p}(G) \subseteq C_{0}(G)$, for a simple element $f=v * u^{\vee}$ of $A_{p}(G)$,

$$
\left\langle\lambda\left(x_{\alpha}\right), v * u^{\vee}\right\rangle=\left\langle\lambda\left(x_{\alpha}\right) u, v\right\rangle=\int_{G} x_{\alpha}^{-1} u(y) v(y) d y=\left(v * u^{\vee}\right)\left(x_{\alpha}\right) \rightarrow 0 .
$$

Hence $T=0$. Let $\Gamma=\{\lambda(x): x \in G\}$. By hypothesis, on $(\Gamma$, weak*) the multiplication is jointly continuous, and the mapping $x \mapsto \lambda(x)$ from $G$ onto 
$\Gamma$ is a homeomorphism. It follows that $\Gamma$ is a group. This being impossible, we conclude that $G$ is compact.

8(c) Invariant subalgebras of $A_{p}(G)$ and compact subgroups of $G$. In this subsection our aim is to show that there exists a one-to-one correspondence between certain closed right translation invariant subalgebras of $A_{p}(G)$ and the class of compact subgroups of $G$.

Let $G$ be a locally compact group. If $\varphi$ is in $A_{p}(G) \quad(1<p<\infty)$ and $a$ in $G$, then, as well known and readily checked, $r_{a} \varphi$ and $l_{a} \varphi$ are also in $A_{p}(G)$ and $\left\|r_{a} \varphi\right\|=\left\|l_{a} \varphi\right\|$. Here $\left(r_{a} \varphi\right)(x)=\varphi(a x)$ and $\left(l_{a} \varphi\right)(x)=\varphi(a x)$. In this section we shall regard $A_{p}(G)$ as a subspace of $L^{\infty}(G)$ and by $\sigma\left(A_{p}(G), L^{1}(G)\right)$ we shall denote the topology induced on $A_{p}(G)$ by the weak*-topology of $L^{\infty}(G)$. To each subgroup $H$ of $G$ we associate the following subspace of $A_{p}(G)$.

$$
V_{p}(H)=\left\{\varphi \in A_{p}(G): l_{h} \varphi=\varphi \text { for all } h \in H\right\} .
$$

Lemma 8.9. For any closed subgroup $H$ of $G$ and $1<p<\infty, V_{p}(H)$ is a $\sigma\left(A_{p}(G), L^{1}(G)\right)$-closed (hence norm closed) right translation invariant subalgebra of $A_{p}(G)$ and is such that, for $\varphi$ in $V_{p}(H), \varphi^{\vee}$ and $\bar{\varphi}$ are also in $V_{p}(H)$. Proof. It is easy to see that the set $V_{p}(H)$ is a subalgebra of $A_{p}(G)$. Also $V_{p}(H)$ is $\sigma\left(A_{p}(G), L^{1}(G)\right)$-closed since $A_{p}(G) \subseteq L^{\infty}(G)$ and left translations are weak*-weak* continuous on $L^{\infty}(G)$. If $a \in G$ and $h \in H$, then $l_{h}\left(r_{a} \varphi\right)=$ $r_{a}\left(l_{h} \varphi\right)=r_{a} \varphi$ for all $\varphi$ in $V_{p}(H)$. Hence $V_{p}(G)$ is right translation invariant. Finally, if $\varphi \in V_{p}(G)$ and $h \in H$, then $\varphi^{\vee} \in A_{p}(G)$ and $l_{h^{-1}} \varphi^{\vee}=\left(l_{h} \varphi\right)^{\vee}=\varphi^{\vee}$ for all $h$ in $H$. Hence $\varphi^{\vee} \in V_{p}(H)$. The last assertion is clear.

Lemma 8.10. If $H$ is a compact subgroup of $G$, then $V_{p}(H) \neq\{0\}$ and for $g \notin H$, there exists $\varphi_{0}$ in $V_{p}(H)$ such that $l_{g} \varphi_{0} \neq \varphi_{0}$.

Proof. Let $\varphi$ be an element of $A_{p}(G)$ such that $\varphi(h)=1$ for all $h$ in $H$. Since the left translation acts continuously on $A_{p}(G)[34$, p. 466] and $H$ is compact, $\left\{l_{h} \varphi: h \in H\right\}$ is a compact subset of $A_{p}(G)$. Let $\Delta$ be the closed convex hull of this set. Then the mapping $(h, \varphi) \mapsto l_{h^{-1}} \varphi$ from $H \times \Delta \rightarrow \Delta$ is a continuous action of $H$ on $\Delta$ endowed with the norm topology. Hence, by Day's Fixed Point Theorem [16], there exists $\psi$ in $\Delta$ such that $l_{h} \psi=\psi$ for all $h$ in $H$. Since $\left(l_{h} \varphi\right)(e)=\varphi(h)=1$ for all $h$ in $H, \psi(e)=1$ also. This proves that $\psi$ is a nonzero element of $V_{p}(H)$, and $V_{p}(H) \neq\{0\}$.

For any $g \notin H, H_{g}$ and $H$ are disjoint compact subsets of $G$. Hence, by the tauberian property of $A_{p}(G)$ [56, p. 221], there exists $\varphi$ in $A_{p}(G)$ such that $\varphi(H)=\{1\}$ and $\varphi(H g)=\{0\}$. Now as above we can find $\psi_{0}$ in the closed convex hull of the $\left\{l_{h} \varphi: h \in H\right\}$ such that $l_{h} \varphi_{0}=\varphi_{0}$ for all $h$ in $H$, i.e., $\varphi_{0}$ is in $V_{p}(H)$. But for each $h$ in $H,\left(l_{h} \varphi\right)(y)=\varphi(h g)=0$. So, $\varphi_{0}(g)=0$. Hence $\left(l_{g} \varphi_{0}\right)(e)=0$. As $\varphi_{0}(e)=1$, we see that $l_{g} \varphi_{0} \neq \varphi_{0}$.

The main result of this subsection is the following theorem.

Theorem 8.11. Let $G$ be a locally compact group and $1<p<\infty$. Then the correspondence $H \rightarrow V_{p}(H)$ defines a one-to-one map from all compact subgroups of $G$ onto all nonzero $\sigma\left(A_{p}(G), L^{1}(G)\right)$-closed right translation invariant subalgebras of $A_{p}(G)$ closed under conjugation.

Proof. By Lemmas 8.9 and 8.10, if $H$ is a compact subgroup of $G$, then $V_{p}(H)$ is a nonzero right translation invariant $\sigma\left(A_{p}(G), L^{1}(G)\right)$-closed subalgebra of 
$A_{p}(G)$ closed under conjugation. Also, if $H_{1} \neq H_{2}$ are two compact subgroups of $G$ and $g \in H_{2} \backslash H_{1}$ (say), then by Lemma 8.10 again, there exists $\varphi_{0}$ in $V_{p}\left(H_{1}\right)$ such that $l_{g} \varphi_{0}=\varphi_{0}$, i.e., $\varphi_{0} \notin V_{p}\left(H_{2}\right)$. This proves that the map $H \rightarrow V_{p}(H)$ is one-to-one.

Conversely, let $V$ be a nonzero, right translation invariant $\sigma\left(A_{p}(G), L^{1}(G)\right)$ closed subalgebra of $A_{p}(G)$ that is closed under conjugation. Let

$$
H=\left\{h \in G: l_{h} \varphi=\varphi \text { and } l_{h} \varphi^{\vee}=\varphi^{\vee} \text { for all } \varphi \in V\right\} .
$$

Then $H$ is a nonempty closed subgroup of $G$. To see that $H$ is compact, let $Q=\{\lambda(g): g \in G\} \cup\{0\}$. Then $Q$, as a subset of $\left(P M_{p}(G)\right.$, weak ${ }^{*}$, is compact and $G$ is homeomorphic to $Q \backslash\{0\}$. Since $H$ is closed in $G$, it suffices to show that zero is not a weak*-cluster point of $\{\lambda(h): h \in H\}$. Indeed, if $\left(\lambda\left(h_{\alpha}\right)\right)$ is a net in $\{\lambda(h): h \in H\}$ converging to zero in the weak*-topology of $P M_{p}(G)$, then, for all $g$ in $G$ and $\varphi$ in $V$,

$$
\left(l_{h_{\alpha}} \varphi\right)(g)=\varphi\left(h_{\alpha} g\right)=\left\langle\varphi, \lambda\left(h_{\alpha}\right) \lambda(g)\right\rangle=\left\langle\varphi \cdot \lambda(g), \lambda\left(h_{\alpha}\right)\right\rangle \rightarrow 0 .
$$

Hence, since $\varphi$ in $V, l_{h_{\alpha}} \varphi(g)=\varphi(g)=0$, for all $g$ in $G$. It follows that $\varphi \equiv 0$, and $V=\{0\}$ in contradiction with the hypothesis that $V$ is nonzero, so $H$ is compact.

It remains to show that $V=V_{p}(H)$. Clearly $V \subseteq V_{p}(H)$. Let $\mathscr{A}$ be the weak*-closure of $V$ in $L^{\infty}(G)$. Then, by assumption on $V, \mathscr{A}$ is a right translation invariant $W^{*}$-subalgebra of $L^{\infty}(G)$. Hence, by Theorem 2 in [63, p. 348], there exists a unique closed subgroup $K$ of $G$ such that $\mathscr{A}=\{f \in$ $L^{\infty}(G): l_{k} f=f$ for all $\left.k \in K\right\}$ and $K=\left\{k \in G: l_{k} f=f\right.$ for all $\left.f \in \mathscr{A}\right\}$, which is the same as the set $\left\{k \in G: l_{k} \varphi=\varphi\right.$ for all $\left.\varphi \in V\right\}$. Hence $H=K$ and $\mathscr{A} \supseteq V_{p}(H)$. Since $V$ is $\sigma\left(A_{p}(G), L^{1}(G)\right)$-closed, $\mathscr{A} \cap A_{p}(G)=V$. Consequently $V=V_{p}(H)$.

Corollary 8.12. Let $G$ be a locally compact group, $1<p<\infty$ and $H$ be a closed subgroup of $G$. Then $V_{p}(H) \neq\{0\}$ if and only if $H$ is compact.

Proof. If $H$ is compact, then, by Lemma 8.10, $V_{p}(H) \neq\{0\}$. Conversely if $V_{p}(H) \neq\{0\}$, then by Lemma 8.9 and Theorem 8.11, there exists a unique compact subgroup $K$ of $G$ such that $V_{p}(H)=V_{p}(K)$ and $K=\left\{g \in G: l_{g} \varphi=\right.$ $\varphi$ for all $\varphi$ in $\left.V_{p}(H)\right\}$. Hence $H \subseteq K$ and $H$, being closed, is compact.

Remark 8.13. (a) If the group $G$ is amenable or $p=2$, then the set $\{\lambda(x)$ : $x \in G\}$ is weak*-dense in $P M_{p}(G)$. It follows in these cases a closed subspace $V$ of $A_{p}(G)$ is a right translation invariant if and only if $V$ is right invariant, i.e., $\varphi \cdot T \in V$ for all $\varphi$ in $V$ and $T$ in $P M_{p}(G)$, where $\langle\varphi \cdot T, S\rangle=\langle\varphi, T S\rangle$ for $S$ in $P M_{p}(G)$.

(b) Theorem 8.11 is a weak generalization of a result of Takesaki-Tatsuma [63, Theorem 9] who proved Theorem 8.11 for $p=2$ and with $\sigma\left(A_{p}(G), L^{1}(G)\right)$ closed replaced by norm closed. However, we do not know for $1<p<\infty$, $p \neq 2$, whether a norm-closed right translation invariant subalgebra of $A_{p}(G)$ that is closed under conjugation is necessarily $\sigma\left(A_{\dot{p}}(G), L^{1}(G)\right)$-closed or not.

8(d) Invariant subalgebras of $P M_{p}(G)$ and closed subgroups of $G$. In the preceding subsection we have seen that there is one-to-one correspondence between certain subalgebras of $A_{p}(G)$ and the class of compact subgroups of $G$. In this section our aim is to show that, when $G$ is amenable, there exists a 
similar correspondence between certain subalgebras of $P M_{p}(G)$ and the class of closed subgroups of $G$.

Let $G$ be a locally compact and $1<p<\infty$. For each closed subset $E$ of $G$, let $N_{E}$ be the weak*-closure of the linear span of $\{\lambda(x): x \in E\}$ in $P M_{p}(G)$. Then, as readily checked, $N_{E}$ is a topologically invariant subspace of $P M_{p}(G)$, i.e., $A_{p}(G) N_{E} \subseteq N_{E}$. As shown by Granirer [32] if $G$ is amenable, then a subspace $X$ of $P M_{p}(G)$ is reflexive and topologically invariant if and only if $X=N_{E}$ for some nonempty finite subset of $E$. The main result of this subsection is an analogue of this result of Granirer. It was proved for $p=2$ (without amenability) by Takesaki-Tatsuma [63, Theorem 6].

We need some notations. For each weak*-closed topologically invariant subspace $N$ of $P M_{p}(G) \quad(1<p<\infty)$, let $\Sigma(N)$ denote the closed subset $\{x \in G: \lambda(x) \in N\}$ of $G$. For each $T$ in $P M_{p}(G)$, let [T] denote the weak*-closed topologically invariant subspace in $P M_{p}(G)$ containing the set $\left\{\varphi \cdot T: \varphi \in A_{p}(G)\right\}$. We shall write $\Sigma[T]$ for $\Sigma([T])$. Note that if $G$ is amenable, then the algebra $A_{p}(G)$ has a bounded approximate identity, and in this case $T$ is in $[T]$.

We recall that the support of an element $T$ in $P M_{p}(G)$, denoted by $\operatorname{supp} T$, is the subset of $G$ characterized by $x \notin \operatorname{supp} T$ if and only if there exists a neighborhood $U$ of $x$ such that $\langle T, \varphi\rangle=0$ for all $\varphi$ in $A_{p}(G)$ with $\operatorname{supp} \varphi \subseteq$ $U$. As one can easily see, $\operatorname{supp} T=\Sigma[T]$. The following lemma can be proved using standard arguments (see the proof of Theorem 40.10 of [42]) and the fact that closed subgroups of an amenable group is a set of spectral synthesis for $A_{p}(G) \quad(1<p<\infty)$ [56, Proposition 19.19]. We can safely omit the details.

Lemma 8.14. Let $G$ be an amenable locally compact group, $1<p<\infty$, and $H$ be a closed subgroup of $G$. Then, for any $T$ in $P M_{p}(G), \operatorname{supp} T \subseteq H$ if and only if $T$ belongs to $N_{H}$.

Given any family $\left(H_{\alpha}\right)_{\alpha \in I}$ of closed subgroups of $G$, let $\bigvee_{\alpha} H_{\alpha}$ denote the smallest closed subgroup of $G$ containing each $H_{\alpha}$. Also, if $\left(N_{\alpha}\right)_{\alpha \in I}$ is a family of weak*-closed topologically invariant subalgebras of $P M_{p}(G)$, we denote by $\bigvee_{\alpha \in I} N_{\alpha}$ the smallest weak*-closed topologically invariant subalgebra of $P M_{p}(G)$ that contains each $N_{\alpha}$. In the theorem below, by $\mathscr{H}$ we shall denote the collection of closed subgroups of the given group $G$ and by $\Omega$ the collection of all weak*-closed topologically invariant subalgebras of $P M_{p}(G)$ such that, for $N$ in $\Omega, \Sigma(N)$ is a subgroup of $G$.

Theorem 8.15. Let $G$ be an amenable locally compact group and $1<p<\infty$. Then the mapping $\Gamma: \mathscr{H} \rightarrow \Omega$ defined by $\Gamma(N)=N_{H}$ is a bijection. Furthermore, for any family $\left(H_{\alpha}\right)_{\alpha \in I}$ in $\mathscr{H}, \Gamma\left(\bigcap_{\alpha} H_{\alpha}\right)=\bigcap_{\alpha} \Gamma\left(H_{\alpha}\right)$ and $\Gamma\left(\bigvee_{\alpha} H_{\alpha}\right)=$ $\mathrm{V}_{\alpha} \Gamma\left(H_{\alpha}\right)$.

Proof. Let $H$ be an element of $\mathscr{H}$. Then $N_{H}$ is a weak*-closed topologically invariant subalgebra of $P M_{p}(G)$ with $\Sigma\left(N_{H}\right)=H$. Hence $\Gamma$ maps $\mathscr{H}$ into $\Omega$. To show that $\Gamma$ is one-to-one, let $H_{1}$ and $H_{2}$ be two distinct elements of $\mathscr{H}$ and let, say, $h_{1}$ be an element of $H_{1} \backslash H_{2}$. Then there exists $\varphi$ in $A_{p}(G)$ such that $\varphi\left(H_{2}\right)=\{0\}$ and $\varphi\left(h_{1}\right)=1$. It follows that $\varphi$ belongs to $\left\{\psi \in A_{p}(G): \psi\left(H_{2}\right)=\{0\}\right\}$ but not to $\left\{\psi \in A_{p}(G): \psi\left(H_{1}\right)=\{0\}\right\}$. Hence $N_{H_{1}} \neq N_{H_{2}}$. To see that $\Gamma$ is onto, let $N$ be an element of $\Omega$. Then $\Sigma(N)=H$ is a closed subgroup of $G$ and $N_{H} \subseteq H$. Also if $T$ is in $N$, then 
$\operatorname{supp} T=\Sigma[T] \subseteq \Sigma(N)=H$. It follows from Lemma 8.14 that $T \in N_{H}$. In particular, $N=N_{H}$.

Since $\Gamma$ preserves inclusion, it is obvious that $\bigvee_{\alpha} N_{H_{\alpha}} \subseteq N_{\bigvee_{\alpha} H_{\alpha}}$. But $N_{\bigvee_{\alpha} H_{\alpha}}$ is the weak*-closure of the linear span of the set $\left\{\lambda(x): x \in \bigvee_{\alpha}^{\alpha} H_{\alpha}\right\}$, and each $\lambda(x)$ is in $\bigvee_{\alpha} N_{H_{\alpha}}$ if $x$ is in $\bigvee_{\alpha} H_{\alpha}$. Hence

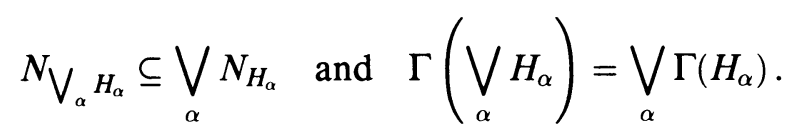

Also, it is obvious that

$$
\bigcap_{\alpha} N H_{\alpha} \supseteq N_{\bigcap_{\alpha} H_{\alpha}}
$$

Now if $T$ is in $\bigcap_{\alpha} N_{H_{\alpha}}$, then supp $T \subseteq H_{\alpha}$ for each $\alpha$ by Lemma 8.14. Hence supp $T \subseteq \bigcap_{\alpha} H_{\alpha}$. Therefore, again by Lemma 8.14, $T$ belongs to $N_{\bigcap_{\alpha} H_{\alpha}}$, and $\Gamma\left(\bigcap_{\alpha} H_{\alpha}\right)=\bigcap_{\alpha} \Gamma\left(H_{\alpha}\right)$.

We do not know if Theorem 8.15 will remain valid when $G$ is not amenable and $p \neq 2$.

\section{ACKNOWLEDGMENT}

The research of the first-named author was supported by an NSERC grant. It was undertaken during the short visit of the second-named author to the Department of Mathematics of Alberta University (Edmonton). He expresses here his sincere thanks to this institution and Professor Anthony To-Ming Lau for the invitation and hospitality. The work of the second-named author is supported by TUBITAK-C2.

\section{REFERENCES}

1. C. Akerman and S. Wright, Compact actions on $C^{*}$-algebras, Glasgow Math. J. 21 (1980), 143-149.

2. R. Arens, The adjoint of a bilinear operation, Proc. Amer. Math. Soc. 2 (1951), 939-948.

3. L. Baggett, A separable group having a discrete dual is compact, J. Funct. Anal. 10 (1972), 131-148.

4. L. Baggett and K. Taylor, Groups with completely reducible regular representations, Proc. Amer. Math. Soc. 72 (1978), 593-600.

5. F. F. Bonsall and J. Duncan, Complete normed algebras, Springer-Verlag, New York and Berlin, 1973.

6. R. Bourgin, Geometric aspects of convex sets with the Radon-Nikodym property, Lecture Notes in Math., vol. 993, Springer-Verlag, 1983.

7. H. Busemann, The geometry of geodesics, Academic Press, New York, 1955.

8. C. Chou, Weakly almost periodic functions and almost convergent functions on a group, Trans. Amer. Math. Soc. 206 (1975), 175-200.

9. __ Minimally weakly almost periodic groups, J. Funct. Anal. 36 (1980), 1-17.

10. C. H. Chu, A note on scattered $C^{*}$-algebras and the Radon-Nikodym property, J. London Math. Soc. 24 (1981), 533-536.

11. __ Von Neumann algebras which are second dual spaces, Proc. Amer. Math. Soc. 112 (1991), 999-1000.

12. C. H. Chu, B. Iochum, and S. Watanabe, $C^{*}$-algebras with the Dunford-Pettis property, Proc. "Function Spaces," Conf. (SIUE, Illinois, 1990), edited by K. Jarosz, Marcel Dekker, New York, Basel and Hong Kong, 1992, pp. 67-70. 
13. C. H. Chu and B. Iochum, The Dunford-Pettis property in $C^{*}$-algebra, Studia Math. 97 (1990), 59-64.

14. P. Civin and B. Yood, The second conjugate space of a Banach algebra as an algebra, Pacific J. Math. 11 (1961), 847-870.

15. M. Cowling, An application of Littlewood-Paley theory in harmonic analysis, Math. Ann. 241 (1979), 83-96.

16. M. M. Day, Correction to my paper "Fixed point theorems for compact convex sets", Illinois J. Math. 8 (1964), 713.

17. F. Delbean, The Dunford-Pettis Theorem for certain uniform algebras, Pacific J. Math. 65 (1976), 29-33.

18. K. Deleeuw and I. Glicksberg, Applications of almost periodic compactifications, Acta Math. 105 (1961), 63-97.

19. J. Diestel, A survey of results related to the Dunford-Pettis property, Contemp. Math., vol. 2, Amer. Math. Soc., Providence, R. I., 1980, pp. 15-60.

20. __ Sequences and series in Banach spaces, Graduate Texts in Math., Springer-Verlag, New York, 1984.

21. J. Diestel and J. J. Uhl, Jr., Vector measures, Math. Surveys, no. 5, Amer. Math. Soc., Providence, R. I., 1977.

22. J. Dixmier, $C^{*}$-algebras, North-Holland, Amsterdam and New York, 1977.

23. J. Duncan and S. A. R. Hosseiniun, The second dual of a Banach algebra, Proc. Roy. Soc. Edinburgh 84A (1979), 309-325.

24. J. Duncan and A. Ülger, Almost periodic functionals on Banach algebras, Rocky Mountain J. Math. 22 (1992), 837-848.

25. P. Eymard, L'algèbre de Fourier d'un groupe localement compact, Bull. Soc. Math. France 92 (1964), 181-236.

26. B. Forrest, Arens regularity and discrete groups, Pacific J. Math. (to appear).

27. B. R. Gelbaum, Tensor product of Banach algebras, Canad. J. Math. 11 (1959), 297-310.

28. N. Ghoussoub, G. Godefroy, B. Maurey and W. Schachermayer, Some topological and geometric structures in Banach spaces, Mem. Amer. Math. Soc., vol. 70, no. 378, 1987.

29. G. Godefroy and B. Iochum, Arens regularity of Banach algebras and the geometry of Banach spaces, J. Funct. Anal. 80 (1988), 47-59.

30. E. Granirer, On group representations whose $C^{*}$-algebras is an ideal in its von Neumann algebra, Ann. Inst. Fourier (4) 29 (1979), 37-52.

31. __ An application of the Radon-Nikodym property in harmonic analysis, Bull. Un. Mat. Ital. B (5) 18 (1981), 663-671.

32. __ Some results on $A_{p}(G)$ submodules of $P M_{p}(G)$, Colloq. Math. 51 (1987), 155-163.

33. $\ldots$ On some space of linear functionals on the algebras $A_{p}(G)$ for locally compact groups, Colloq. Math. 52 (1987), 119-132.

34. E. Granirer and M. Leinert, On some topologies which coincide on the unit sphere of the Fourier-Stieltjes algebra $B(G)$ and of the measure algebra $M(G)$, Rocky Mountain J. Math. 11 (1981), 459-472.

35. W. L. Green and A. T. Lau, Strongly finite von Neumann algebras, Math. Scand. 40 (1977), 105-112.

36. F. Greenleaf, Invariant means on topological groups and their application, Van Nostrand, New York, 1969.

37. A. Grothendieck, Sur les applications linéaires faiblement compactes d'espaces du type $C(K)$, Canad. J. Math. 5 (1953), 129-173.

38. U. Haagerup, The Grothendieck inequality for bilinear forms on $C^{*}$-algebras, Adv. in Math. 56 (1985), 93-116.

39. M. Hamana, On linear topological properties of some $C^{*}$-algebras, Tôhoku Math. J. 29 (1977), 157-163. 
40. G. Hansel and J. P. Troallic, On a class of weakly almost periodic mappings, Semigroup Forum 41 (1990), 357-372.

41. R. E. Harrell and L. A. Karlovitz, Geometry of flat Banach spaces, Trans. Amer. Math. Soc. 192 (1974), 209-218.

42. E. Hewitt and K. Ross, Abstract harmonic analysis, Vols. I and II, Springer-Verlag, New York and Berlin, 1973, 1970.

43. H. E. Jensen, Scattered $C^{*}$-algebras, Math. Scand. 4 (1977), 308-314.

44. E. Kaniuth, Die Structur der regulären Darstellung lokalcompakte Gruppen mit invarianter Umgebungsbasis der Eins, Math. Ann. 194 (1971), 225-248.

45. __ Der typ der regulären Darstellung diskreter Gruppen, Math. Ann. 182 (1969), 334-339.

46. J. L. Kelley, I. Namioka, et al., Linear topological spaces, Van Nostrand, Princeton, N. J., 1963.

47. H. E. Lacey, The isometric theory of classical Banach spaces, Springer-Verlag, New York and Berlin, 1974.

48. A. T. Lau, Closed convex invariant subsets of $L^{p}(G)$, Trans. Amer. Math. Soc. 232 (1977), 131-142.

49. __ , $W^{*}$-algebras and invariant functionals, Studia Math. 56 (1976), 253-261.

50. A. T. Lau and P. F. Mah, Normal structure in dual Banach spaces associated to locally compact groups, Trans. Amer. Math. Soc. 310 (1988), 341-353.

51. A. T. Lau and J. C. S. Wong, Weakly almost periodic elements in $L^{\infty}(G)$ of a locally compact group, Proc. Amer. Math. Soc. 107 (1989), 1031-1036.

52. K. McKennon, Multipliers, positive functionals, positive-definite functions and FourierStieltjes transforms, Mem. Amer. Math. Soc. 111 (1971).

53. T. W. Palmer, Classes of nonabelian, noncompact locally compact groups, Rocky Mountain J. Math. 8 (1973), 683-741.

54. A. Paterson, Amenability, Math. Surveys and Monographs, vol. 29, Amer. Math. Soc., Providence, R. I., 1988.

55. A. Pelczynksi and Z. Semedani, Spaces of continuous functions. III, Studia Math. 18 (1959), 213-222.

56. J. P. Pier, Amenable locally compact groups, Wiley, New York, 1984.

57. H. Rosenthal, A characterization of Banach spaces containing $l^{1}$, Proc. Nat. Acad. Sci. U.S.A. 71 (1974), 2411-2413.

58. R. A. Ryan, The Dunford-Pettis property and projective tensor products, Bull. Polish Acad. Sci. 35 (1987), 785-792.

59. K. Saito, On the preduals of $W^{*}$-algebras, Tôhoku Math. J. 19 (1967), 324-331.

60. S. Sakai, $C^{*}$-algebras and $W^{*}$-algebras, Springer-Verlag, New York and Berlin, 1971.

61. M. Smith, Regular representation of discrete groups, J. Funct. Anal. 11 (1972), 401-406.

62. M. Takesaki, Theory of operator algebras. I, Springer-Verlag, New York and Berlin, 1979.

63. M. Takesaki and N. Tatsuma, Duality and subgroups, Ann. of Math. 93 (1971), 344-364.

64. __, Duality and subgroups. II, J. Funct. Anal. 11 (1972), 182-190.

65. K. Taylor, The type structure of the regular representation of a locally compact group, Math. Ann. 222 (1976), 211-214.

66. Geometry of the Fourier algebras and locally compact groups with atomic representations, Math. Ann. 262 (1983), 183-190.

67. E. Thoma, Ein charakterisierung diskrèter gruppen von typ I, Invent. Math. 6 (1968), 190196.

68. J. Tomiyama, Tensor product of commutative Banach algebras, Tôhoku Math. J. 12 (1960), 147-154.

69. A. Ülger, Arens regularity sometimes implies the RNP, Pacific J. Math. 143 (1990), 377-399. 70. $\frac{}{704}$, Weakly bilinear forms and Arens regularity, Proc. Amer. Math. Soc. 101 (1987), 697- 
71. _ Arens regularity of the algebra $A \hat{\otimes} B$, Trans. Amer. Math. Soc. 305 (1988), 623-639.

72. __ Arens regularity of the algebra $K(X)$, Monatsh. Math. 105 (1988), 313-318.

73. P. S. Wang, On isolated points in the dual spaces of locally compact group, Math. Ann. 218 (1975), 19-34.

74. F. Yeadon, A new proof of the existence of a trace in a finite von Neumann algebra, Bull. Amer. Math. Soc. 77 (1971), 257-260.

75. N. J. Young, Periodicity of functionals and representations of normed algebras on reflexive spaces, Proc. Edinburgh Math. Soc. 20 (1976), 99-120.

Department of Mathematics, University of Alberta, Edmonton, T6G 2G1, Canada E-mail address: usertlau@ualtamts.bitnet

Department of Mathematics, Bö̈aziç University, 80815 Bebek-Istanbul, Turkey Current address: Department of Mathematics, Bilkent University, 06533 Ankara, Turkey E-mail address: userulger@trbilun.bitnet 